IMA Journal of Numerical Analysis (2012) Page 1 of 24

doi:10.1093/imanum/drnxxx

\title{
Localized adaptive radiation condition for coupling boundary with finite element methods applied to wave propagation problems
}

\author{
ABDERRAHMANE BENDALI $\dagger$, \\ University of Toulouse, \\ INSA de Toulouse, Institut Mathématique de Toulouse, UMR CNRS 5219, \\ 135 avenue de Rangueil F31077, Toulouse cedex 1, France, and \\ CERFACS \\ 42 Avenue Gaspard Coriolis, 31057 Toulouse Cedex 01, France, \\ AND \\ YACINE BOUBENDIR † \\ New Jersey, Institute of Technology, \\ Department of Mathematical Sciences and Center for Applied Mathematics and Statistics, \\ NJIT, Univ. Heights. 323 Dr. M. L. King Jr. Blvd, Newark, NJ 07102, USA, \\ AND \\ NICOLAS ZERBIB \\ ESI Group, \\ 20 rue du Fonds Pernant, 60471 COMPIEGNE Cedex, France.
}

[Received on ; revised on ]

\begin{abstract}
The wave propagation problems addressed in this paper involve a relatively large and impenetrable surface on which is posed a comparatively small penetrable heterogeneous material. Typically the numerical solution of such kinds of problems is solved by coupling boundary and finite element methods. However, a straightforward application of this technique gives rise to some difficulties which mainly are related to the solution of a large linear system whose matrix consists of sparse and dense blocks. To face such difficulties, the adaptive radiation condition technique is modified by localizing the truncation interface only around the heterogeneous material. Stability and error estimates are established for the underlying approximation scheme. Some alternative methods are recalled or designed making it possible to compare the numerical efficiency of the proposed approach.
\end{abstract}

Keywords: Helmholtz equation, domain decomposition methods, finite element methods, boundary element method.

\section{Introduction}

Coupling dissimilar numerical schemes generally enhances the solution process but may lead to specific difficulties. For instance, such a coupling is particularly adapted for dealing with the radiation or the scattering of time-harmonic waves when the problem involves heterogeneous material. The boundary el-

\footnotetext{
†Email: abendali@insa-toulouse.fr

‡Corresponding author. Email: yassine.boubendir@njit.edu

${ }^{\S}$ Email: nicolas.zerbib@esi-group.com
}

(c) The author 2012. Published by Oxford University Press on behalf of the Institute of Mathematics and its Applications. All rights reserved. 
ement method (BEM) efficiently handles the truncation of the solution domain as a transparent radiation condition, whereas the zones where the material presents varying characteristics are approximated by a finite element method (FEM) (cf., e.g., Johnson \& Nédélec (1980); Costabel (1988); Bendali \& Fares (2008)). However a straightforward coupling of the two methods leads to a linear system, generally of very large scale, with a matrix partly sparse and partly dense. This kind of linear system is difficult to solve, especially when the computation is performed on a parallel platform (Boubendir et al. (2008)). Domain decomposition methods (DDMs) display distinct advantages for overcoming such difficulties. Indeed, non-overlapping DDMs were successfully applied for the combination of a FEM with a BEM to solve the Helmholtz equation (Boubendir et al. (2008); Bendali \& Fares (2008); Bendali et al. (2007)). However, the wave problems treated in these papers concern obstacles where the heterogeneous region completely encloses the impenetrable boundary.

In this work, we are interested in impenetrable surfaces with relatively large size on which a heterogeneous object of relatively small size is posed. A widespread instance of such a problem is met in the numerical simulation of the radiation of an antenna posed on a large metallic structure. In this case, the non-overlapping domain decomposition developed in Bendali et al. (2007) and mentioned above is not efficient any more mainly because of the slow convergence of the underlying iterative method. In this work, we propose an alternative method derived from a modification of the adaptive radiation condition approach (Jin (2002); Alfonzetti et al. (1998); Li \& Cendes (1995)). In its standard form, this technique consists of enclosing the computational domain by an artificial truncating surface on which the adaptive radiation condition is posed. This condition is expressed using integral operators acting as a correction term of the absorbing boundary condition. Precisely, the more iterations performed, the closer this condition converges towards the transparent one. However, enclosing completely the computational domain by an artificial surface in this range results in a too large size problem. Even worse, approximating the propagation of a wave along large distances by a FEM may be very problematic because of the dispersion errors, which can severely damage the accuracy of the final result. The difference with the method we propose resides in the localization of the truncating interface only around the heterogenous region (Zerbib (2006)). This truncation generates a relatively small bounded domain dealt with by a FEM, and suitably coupled with a BEM expressing the solution on the impenetrable surface. The resulting formulation, based on a particular overlapping domain decomposition method, is solved iteratively where finite and boundary element linear systems are solved separately. This way to proceed can hence be called a localized adaptive radiation condition.

The main focus of this paper is on the numerical analysis of the FEM-BEM coupling. We establish its well-posedeness and stability using Fredholm alternative, and the theory of mixed formulations (Brezzi \& Fortin (1991)). In addition to this analysis, we propose alternative methods based mainly on non-overlapping domain decomposition methods. The goal is to demonstrate that the proposed approach performs well in comparison with these competing methods. Since this study is of methodological interest, for the sake of simplicity we limit ourselves in this paper to the two-dimensional case. The extension to $3 \mathrm{D}$ problems governed by the Helmholtz equation does not add any difficulty except a more intricate notation. However, this is not the case with Maxwell system, for which an application of this technique is by no means straightforward. The description of the related algorithm and its implementation on a parallel platform will be given in a forthcoming paper.

This paper is organized as follows. Section 2 consists of a description of a model problem and its appropriate functional setting. Section 3 is devoted to the development of the localized adaptive radiation condition. In section 4, we begin by introducing the FEM-BEM coupling formulation and proving its stability and convergence. Then, we detail its numerical implementation, and more particularly, how suitably the iterative procedure avoids solving a linear system with a matrix partly sparse and partly 


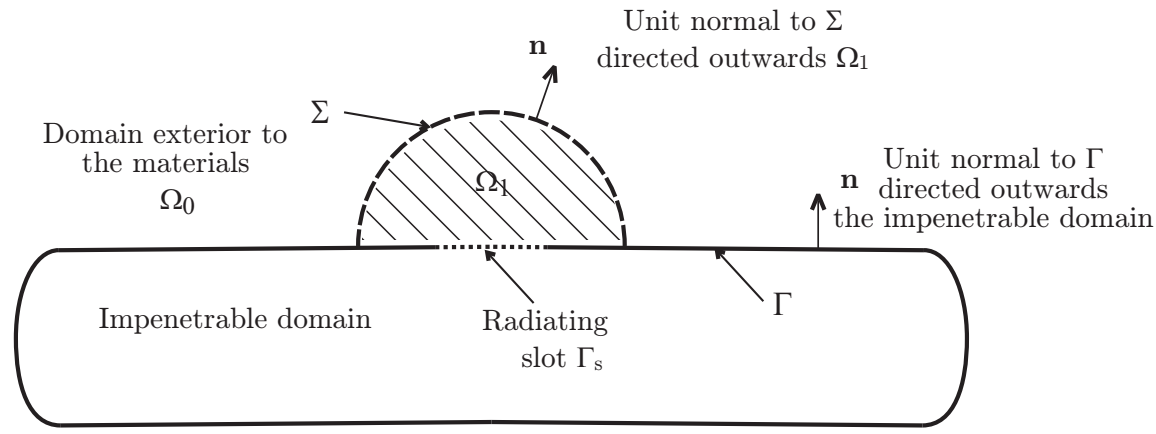

FIG. 1. Non-overlapping decomposition of the exterior domain $\Omega$ into $\Omega_{0}$ and $\Omega_{1}$.

dense. Finally in section 5, we show how to use domain decomposition principles to develop alternative numerical procedures. Experiments validating the localized adaptive radiation as well as comparing its performances with the other competing methods are next presented. Section 6 is reserved for some concluding remarks.

\section{The model problem}

We are dealing in this paper with wave problems for which the heterogeneous media extends in a relatively small part of the propagation domain. Precisely, we consider the geometrical data depicted in Fig. 1. The model problem we are looking at can be stated as follows

$$
\left\{\begin{array}{l}
\nabla \cdot(\chi \nabla u)+\chi \kappa^{2} n^{2} u=0 \quad \text { in } \Omega, \\
\chi \partial_{\mathbf{n}} u=-f \text { on } \Gamma \\
\lim _{|x| \rightarrow \infty}|x|^{1 / 2}\left(\partial_{|x|} u-i \kappa u\right)=0
\end{array}\right.
$$

where $\Omega$ is the complement of the impenetrable obstacle. We indicate by $\Omega_{1}$ a bounded domain filled by a possibly heterogeneous material and posed on a slot $\Gamma_{\text {slot }}$. This slot is also part of the boundary $\Gamma$ of the impenetrable obstacle on which are applied the sources producing the radiated wave $u$. As a result, the Neumann data $f$ are zero on all of $\Gamma$ except on $\Gamma_{\text {slot }}$. The interface $\Sigma$ separates $\Omega_{1}$ from the free propagation domain $\Omega_{0}$, characterized by the wave number $\kappa>0$. It is worth noting that $\Omega_{0}$ and $\Omega_{1}$ constitute a non-overlapping decomposition of $\Omega$. According to the context, $\mathbf{n}$ will denote the normal to $\Gamma$ or to $\Sigma$ directed outwards respectively the impenetrable obstacle enclosed by $\Gamma$ or the domain $\Omega_{1}$ (see Figure 1 for the geometric configuration of the problem).

The fact that the heterogeneous medium is confined to $\Omega_{1}$ is expressed by assuming that the possibly varying contrast coefficients satisfy $\chi=n=1$ in $\Omega_{0}$.

It will be convenient to express problem (2.1) in the form of the following system consisting of two boundary-value problems

$$
\left\{\begin{array}{l}
\Delta u_{0}+\kappa^{2} u_{0}=0 \text { in } \Omega_{0} \\
\partial_{\mathbf{n}} u_{0}=0 \text { on } \Gamma \cap \partial \Omega_{0} \\
\lim _{|x| \rightarrow \infty}|x|^{1 / 2}\left(\partial_{|x|} u_{0}-i \kappa u_{0}\right)=0
\end{array}\right.
$$




$$
\left\{\begin{array}{l}
\nabla \cdot\left(\chi \nabla u_{1}\right)+\chi \kappa^{2} n^{2} u_{1}=0 \text { in } \Omega_{1}, \\
\chi \partial_{\mathbf{n}} u_{1}=-f \text { on } \Gamma \cap \partial \Omega_{1},
\end{array}\right.
$$

coupled by means of the following transmission conditions on $\Sigma$

$$
u_{0}=u_{1}, \quad \partial_{\mathbf{n}} u_{0}=\chi \partial_{\mathbf{n}} u_{1} .
$$

Passing from one to the other formulation simply amounts to writing $u_{0}=\left.u\right|_{\Omega_{0}}, u_{1}=\left.u\right|_{\Omega_{1}}$.

Model problem (2.1) is given in the general framework of time-harmonic wave propagation. In the two-dimensional electromagnetic case, the variables $\chi$ and $n$ can be expressed by means of the relative dielectric permittivity and the relative magnetic permeability. The wave $u$ represents the component of the magnetic field normal to the plane of propagation. For acoustics, the variable $\chi$ is related to the fluid density, and $u$ can be either the potential of the velocity or the pressure. For both cases, $n$ is the refractive index in $\Omega_{1}$. Usual energy considerations and physical properties of penetrable media ensure that the following bounds

$$
0<\chi_{*} \leqslant \mathfrak{R} \chi \leqslant \chi^{*},-\chi^{*} \leqslant \mathfrak{I} \chi \leqslant 0,1 \leqslant \Re n \leqslant n^{*}, 0 \leqslant \mathfrak{I} n \leqslant n^{*}
$$

hold true almost everywhere on $\Omega_{1}$. We denoted by $\mathfrak{R} z$ and $\mathfrak{I} z$ the real and imaginary parts respectively of the complex number $z$.

All the considered domains are supposed lipschitzian (cf., e.g., McLean (2000)) with a boundary being moreover piecewise $\mathscr{C}^{\infty}$. Notation and functional spaces, including Sobolev spaces, which are standard in the partial differential equations theory are used without further comment (cf., e.g., Taylor (1996); McLean (2000)).

The sources $f$ are assumed to be in $\widetilde{H}^{-1 / 2}\left(\Gamma_{\text {slot }}\right)$, i.e., $f \in H^{-1 / 2}(\Gamma)$ and is zero outside $\Gamma_{\text {slot }}$ (cf. McLean (2000)). Adapting, for example, the techniques used in Wilcox (1975) for the usual Helmholtz equation, we readily get that problem (2.1) admits one and only one solution $u$ in the setting of the following Fréchet space

$$
H_{\mathrm{loc}}^{1}(\bar{\Omega})=\left\{v \in \mathscr{D}^{\prime}(\Omega) ; \varphi v \in H^{1}(\Omega), \forall \varphi \in \mathscr{D}\left(\mathbb{R}^{2}\right)\right\}
$$

\section{The adaptive localized radiation condition}

\subsection{The derivation of the FEM-BEM coupling procedure}

The method we propose resembles in its principle the one developed in Lenoir \& Jami (1978) for the Laplace equation and subsequently extended to Maxwell's equations in Hazard \& Lenoir (1996). The standard method consists of enclosing completely the obstacle (here meaning the complement of the free propagation zone $\Omega_{0}$ ) using a fictitious boundary $S$. The step following this truncation resides in approximating the problem by a combination of FEM and BEM where the integral equations are defined on $S$ but with densities on the boundary $\partial \Omega_{0}$, sometimes conveniently denoted $\Gamma_{\Sigma}$ below. These formulations require an explicit expression of the variable $p=\left.\partial_{\mathbf{n}} u_{0}\right|_{\Sigma}$ which is not available in the framework of an approximation of the problem in the domain enclosed by $S$ by a nodal finite FEM. Actually in Lenoir \& Jami (1978), $p$ is a given datum, and in Liu \& Jin (2001), the authors resort to a formulation whose unknowns are the electric and magnetic fields respectively inside and outside $\Omega_{1}$. In the present context, the approach in Liu \& Jin (2001) would have consisted in using a usual nodal FEM outside $\Omega_{1}$ combined with a mixed one in $\Omega_{1}$ (see, e.g., Brezzi \& Fortin (1991) for the 


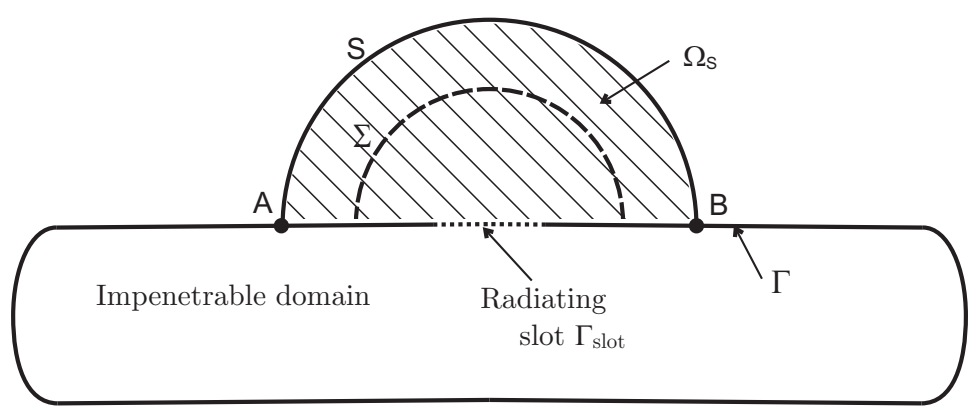

FIG. 2. The bounded domain $\Omega_{S}$ and the fictitious boundary $S$ on which is posed the adaptive radiation condition.

solution of second-order elliptic boundary-value problems by mixed FEMs). The method considered below overcomes this difficulty.

The approach we propose in this work consists in localizing the truncation only around the penetrable material. This is performed, as depicted in Fig. 2, by introducing a fictitious boundary $S$ which in turn produces the bounded domain $\Omega_{S}$ limited by $S$ and the impenetrable zone. It is worth mentioning that $\Omega_{S}$ contains the domain $\Omega_{1}$ in which the contrast coefficient $\chi$ and the refracting index may be not set to 1 .

The goal is to derive a formulation of problem (2.1) as a coupled system composed of two equations with two unknowns $u_{0}$ and $u_{S}$ where the function $u_{S}=\left.u\right|_{\Omega_{S}}$ is approximated by a FEM, and $u_{0}$, already defined in the above section, is computed using an integral equation on $\Gamma_{\Sigma}$. The treatment adopted for $u_{0}$ is based on an integral representation of this function in terms of a single- and a double-layer potential created by densities on $\Gamma_{\Sigma}$, and as a result can be seen as the restriction to $\Omega_{0}$ of the solution of a transmission problem posed on all of the plane $\mathbb{R}^{2}$ (cf., e.g., Hsiao \& Wendland (2008); Nédélec (2001); McLean (2000)). In view of the equations that are set in $\Omega_{S}$, we are in the case of a particular decomposition with an overlap of the computational domain (see similar ideas in Ben Belgacem et al. (2009); Ben Belgacem et al. (2005) for the usual adaptive radiation condition). However, as will be clear below, it will be more convenient not to distinguish $u_{0}$ from $u_{S}$ and to refer to them as the same function $u$ in $H_{\mathrm{loc}}^{1}(\bar{\Omega})$.

Simply by restricting $u$ to $\Omega_{S}$, we get from (2.1) that $u$ satisfies

$$
\left\{\begin{array}{l}
\nabla \cdot(\chi \nabla u)+\chi \kappa^{2} n^{2} u=0, \quad \text { in } \Omega_{S}, \\
\chi \partial_{\mathbf{n}} u=-f \quad \text { on } \Gamma \cap \partial \Omega_{S} .
\end{array}\right.
$$

In $\Omega_{0}$, we use the integral representations of the solutions to the Helmholtz equation satisfying the Sommerfeld radiation condition (cf., e.g., Nédélec (2001); Colton \& Kress (1983, 1992); Bendali \& Fares (2008))

$$
u(x)=V^{*, \Sigma} p(x)-N^{*, \Gamma_{\Sigma}} u(x), \quad x \in \Omega_{0},
$$

with

$$
\begin{aligned}
V^{*, \Sigma} p(x) & =\int_{\Sigma} G(x, y) p(y) d s_{y} \\
p & =-\left.\chi \partial_{\mathbf{n}} u\right|_{\Sigma}
\end{aligned}
$$




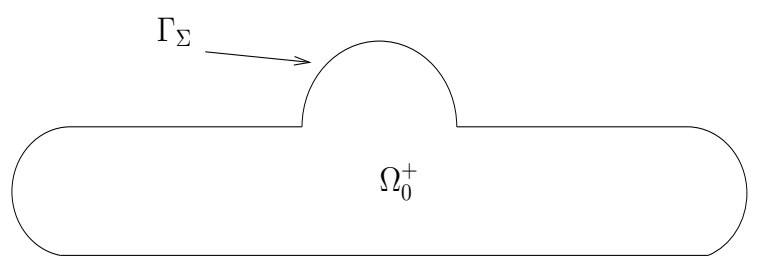

FIG. 3. Representation of $\Omega_{0}^{+}$and its boundary $\Gamma_{\Sigma}$.

$$
N^{*, \Gamma_{\Sigma}} u(x)=-\int_{\Gamma_{\Sigma}} \partial_{n_{y}} G(x, y) u(y) d s_{y}
$$

where the kernel is expressed in terms of the Hankel function of the first kind of order 0 by

$$
G(x, y)=\frac{i}{4} H_{0}^{(1)}(\kappa|x-y|), \quad x \neq y \in \mathbb{R}^{2} .
$$

In the sequel, the context indicates the curve to which refers the arc length $s$.

The determination of $u$ is therefore reduced to its traces $p$ and $\left.u\right|_{\Gamma_{\Sigma}}$. Observe that transmission conditions (2.4) define these traces unambiguously. Moreover, it is clear in (3.2) that the involved values of $u$ are those corresponding to its trace $\left.u\right|_{\Gamma_{\Sigma}}$ avoiding the need of an explicit notation for the restriction. Finally, it is worth mentioning that integral (3.2) vanishes for $x \in \Omega_{0}^{+}$, the domain enclosed by $\Gamma_{\Sigma}$ (Figure 3).

Problem (3.1), once solved, determines $u$ on $\Omega_{S}$ only. The complete determination of $u$ on $\Gamma_{\Sigma}$ is obtained by writing an adequate integral equation outside $\Omega_{S}$ using the condition $\partial_{\mathbf{n}} u=0$ on $\Gamma$, thus yielding the FEM-BEM coupling approach.

REMARK 3.1 In the method of Jami-Lenoir (Lenoir \& Jami (1978)), the boundary $\Sigma$ is contained in the interior of $\Omega_{S}$ so that $u$ can be obtained outside $\Omega_{S}$ by an explicit integral representation. This is in contrast with the need here to solve an integral equation on a part of $\Gamma$.

The derivation of the FEM-BEM coupling procedure can be introduced starting from the following Green formula

$$
\int_{\Omega_{S}} \chi\left(\nabla u \cdot \nabla v-\kappa^{2} n^{2} u v\right) d x=\left\langle\partial_{\mathbf{n}} u, v\right\rangle_{\widetilde{H}^{-1 / 2}(S), H^{1 / 2}(S)}+\langle f, v\rangle_{\widetilde{H}^{-1 / 2}\left(\Gamma_{\text {slot }}\right), H^{1 / 2}\left(\Gamma_{\text {slot }}\right)}
$$

where $\langle\cdot, \cdot\rangle_{\widetilde{H}^{-1 / 2}(S), H^{1 / 2}(S)}$ denotes the duality pairing between $\widetilde{H}^{-1 / 2}(S)$ and $H^{1 / 2}(S)$, and $v$ is an arbitrary test function in $H_{\text {loc }}^{1}(\bar{\Omega})$. The space $\widetilde{H}^{-1 / 2}(S)$ is defined similarly to $\widetilde{H}^{-1 / 2}\left(\Gamma_{\text {slot }}\right)$.

The adaptive approach consists mainly in solving problem (3.7) by means of an iterative method, the term $\partial_{\mathbf{n}} u$ at the right-hand side being assumed to be known before each iteration and updated after terminating it. However, in this form, there is no garantee that problem (3.7) can be safely solved. Usually the stabilization term $-i \kappa \int_{S} u v d s$, corresponding to the crudest absorbing radiation condition, is appended to both sides of (3.7) to alleviate this difficulty. However, $S$ is here an open curve having its end-points $A$ and $B$ on $\Gamma$ (see Fig. 2). To avoid singular integrals near these points, we introduce a cut-off function $\eta \in \mathscr{D}\left(\mathbb{R}^{2}\right)$ such that $0 \leqslant \eta \leqslant 1, \eta=1$ on $S$, except small neighborhood of any of $A$ and $B, \eta$ being moreover equal to 0 around $A$ and $B$, and write (3.7) in the following form

$$
\begin{aligned}
\int_{\Omega_{S}} \chi\left(\nabla u \cdot \nabla v-\kappa^{2} n^{2} u v\right) d x-i \kappa \int_{S} \eta u v d s & =\left\langle\partial_{\mathbf{n}} u, v\right\rangle_{\widetilde{H}^{-1 / 2}(S), H^{1 / 2}(S)} \\
& -i \kappa \int_{S} \eta u v d s+\langle f, v\rangle_{\widetilde{H}^{-1 / 2}\left(\Gamma_{\text {slot }}\right), H^{1 / 2}\left(\Gamma_{\text {slot }}\right)}
\end{aligned}
$$


To complete the coupling procedure, we then consider the curve $\Gamma_{S}$ obtained by joining $S$ and the part of $\Gamma$ outside $\Omega_{S}$ and note that we can express the fact that $\partial_{\mathbf{n}} u=0$ there outside $S$ variationally as follows

$$
\left\langle\partial_{\mathbf{n}} u, v\right\rangle_{\widetilde{H}^{-1 / 2}(S), H^{1 / 2}(S)}=\left\langle\partial_{\mathbf{n}} u, v\right\rangle_{H^{-1 / 2}\left(\Gamma_{S}\right), H^{1 / 2}\left(\Gamma_{S}\right)},
$$

for all test function $v$. We thus arrive to

$$
\begin{aligned}
\int_{\Omega_{S}} \chi & \left(\nabla u \cdot \nabla v-\kappa^{2} n^{2} u v\right) d x-i \kappa \int_{S} \eta u v d s= \\
& \left\langle\partial_{\mathbf{n}} u, v\right\rangle_{H^{-1 / 2}\left(\Gamma_{S}\right), H^{1 / 2}\left(\Gamma_{S}\right)}-i \kappa \int_{S} \eta u v d s+\langle f, v\rangle_{\widetilde{H}^{-1 / 2}\left(\Gamma_{\text {slot }}\right), H^{1 / 2}\left(\Gamma_{\text {slot }}\right)}
\end{aligned}
$$

where the traces in the right-hand side are expressed from the integral representation (3.2) of $u$

$$
\left\{\begin{array}{l}
\left.\eta u\right|_{S}=\eta V^{S, \Sigma} p-\eta N^{S, \Gamma_{\Sigma}} u \\
\left.\partial_{\mathbf{n}} u\right|_{\Gamma_{S}}=\partial_{\mathbf{n}} V^{\Gamma_{S}, \Sigma} p-\partial_{\mathbf{n}} N^{\Gamma_{S}, \Gamma_{\Sigma}} u
\end{array}\right.
$$

Clearly, since $\Sigma$ and $S$ share no common point and $\eta$ is zero in the proximity of the end-points of $S$, if $p$ and $u$ are sufficiently smooth functions, say for example continuous, only the integral corresponding to $\partial_{\mathbf{n}} N^{*, \Gamma_{\Sigma}} u$ in (3.11) is an improper integral which can be expressed by means of a weakly singular kernel as follows

$$
\begin{aligned}
& \left\langle\partial_{\mathbf{n}} N^{\Gamma_{S}, \Gamma_{\Sigma}} u, v\right\rangle_{H^{-1 / 2}\left(\Gamma_{S}\right), H^{1 / 2}\left(\Gamma_{S}\right)}= \\
& \quad\left\langle\partial_{S} v, V^{\Gamma_{S}, \Gamma_{\Sigma}} \partial_{S} u\right\rangle_{H^{-1 / 2}\left(\Gamma_{S}\right), H^{1 / 2}\left(\Gamma_{S}\right)}-\kappa^{2}\left\langle v \tau, V^{\Gamma_{S}, \Gamma_{\Sigma}}(u \tau)\right\rangle_{H^{-1 / 2}\left(\Gamma_{S}\right), H^{1 / 2}\left(\Gamma_{S}\right)}
\end{aligned}
$$

from a slight adaptation of the case where $\Gamma_{S}=\Gamma_{\Sigma}$ (cf., e.g., (Hsiao \& Wendland, 2008, p. 5)). The superscripts in the integral operators indicate that they correspond to a potential created by a density on $\Gamma_{\Sigma}$ and evaluated on $\Gamma_{S}$, and $\tau$ is the unit tangent vector pointing in the growth direction of the arc length $s$.

As indicated above, we need to deal with (3.4) in order to be able to use a nodal approximation of (3.10). We use a standard technique for gluing finite element approximations of different kinds or associated with non-conforming meshes generally called mortar FEM (cf., e.g., Ben Belgacem (1999)). It is worth mentioning that here only standard meshes and finite element methods of the same kind are used. This way to proceed is just considered as a tool providing an approximation for the additional unknown $p$ in the framework of a nodal finite elemnt method. This technique consists in breaking the continuity across $\Sigma$ that $u$ is compelled to satisfy a priori and to express it as a constraint. The Lagrange multiplier corresponding to this constraint will be precisely the unknown $p$. It is hence more convenient to denote by separate symbols: $u_{0}$ for the restriction of $u$ to $\Omega_{0} \cap \Omega_{S}$ and $\Gamma_{\Sigma}$ and $u_{1}$ for its restriction to $\Omega_{1}$. More precisely, we will use the following functional framework

$$
\left\{\begin{array}{c}
X_{0}=\left\{u_{0} \text { defined (a.e.) on } \Omega_{0} \cap \Omega_{S} \text { and } \Gamma_{\Sigma} ;\right. \\
\left.\quad \exists U \in H^{1}\left(\Omega_{0}\right),\left.U\right|_{\Gamma_{\Sigma}}=\left.u_{0}\right|_{\Gamma_{\Sigma}} \text { and }\left.U\right|_{\Omega_{0} \cap \Omega_{S}}=\left.u_{0}\right|_{\Omega_{0} \cap \Omega_{S}}\right\} \\
X_{1}=H^{1}\left(\Omega_{1}\right), \quad X=X_{0} \times X_{1},
\end{array}\right.
$$

relation (3.4) and (3.10) to write

$$
\begin{aligned}
\int_{\Omega_{S} \cap \Omega_{0}}\left(\nabla u_{0} \cdot \nabla v_{0}-\kappa^{2} n^{2} u_{0} v_{0}\right) d x-i \kappa \int_{S} \eta u_{0} v d s \\
+\int_{\Omega_{1}} \chi\left(\nabla u_{1} \cdot \nabla v_{1}-\kappa^{2} n^{2} u_{1} v_{1}\right) d x+\left\langle p, v_{1}-v_{0}\right\rangle_{\widetilde{H}^{-1 / 2}(\Sigma), H^{1 / 2}(\Sigma)}= \\
\quad\left\langle\partial_{\mathbf{n}} u, v_{0}\right\rangle_{H^{-1 / 2}\left(\Gamma_{S}\right), H^{1 / 2}\left(\Gamma_{S}\right)}-i \kappa \int_{S} \eta u v_{0} d s+\left\langle f, v_{1}\right\rangle_{\widetilde{H}^{-1 / 2}\left(\Gamma_{\text {slot }}\right), H^{1 / 2}\left(\Gamma_{\text {slot }}\right)}
\end{aligned}
$$


for all $\left(v_{0}, v_{1}\right) \in X$. Using then the integral represensation of $\left.\partial_{\mathbf{n}} u\right|_{\Gamma_{S}}$ and $\left.u\right|_{S}$ given above in (3.11), we readily arrive to the formulation effectively used to solve problem (2.1) numerically

$$
\left\{\begin{array}{l}
(u, p) \in X \times M, \forall(v, q) \in X \times M \\
a(u, v)+d\left(u_{0}, v_{0}\right)+b(p, v)+r\left(p, v_{0}\right)=\left\langle f, v_{1}\right\rangle_{\widetilde{H}^{-1 / 2}\left(\Gamma_{\text {slot }}\right), H^{1 / 2}\left(\Gamma_{\text {slot }}\right)} \\
b(q, u)=0
\end{array}\right.
$$

with the following notation

$$
\left\{\begin{array}{l}
a_{0}\left(u_{0}, v_{0}\right)=\int_{\Omega_{S} \cap \Omega_{0}}\left(\nabla u_{0} \cdot \nabla v_{0}-\kappa^{2} u_{0} v_{0}\right) d x-i \kappa \int_{S} \eta u_{0} v_{0} d s \\
a_{1}\left(u_{1}, v_{1}\right)=\int_{\Omega_{1}} \chi\left(\nabla u_{1} \cdot \nabla v_{1}-\kappa^{2} n^{2} u_{1} v_{1}\right) d x \\
a(u, v)=a_{0}\left(u_{0}, v_{0}\right)+a_{1}\left(u_{1}, v_{1}\right) \\
d\left(u_{0}, v_{0}\right)=\left\langle\partial_{\mathbf{n}} N^{\Gamma_{S}, \Gamma_{\Sigma}} u_{0}, v_{0}\right\rangle_{H^{-1 / 2}\left(\Gamma_{S}\right), H^{1 / 2}\left(\Gamma_{S}\right)}-i \kappa \int_{S} \eta v_{0} N^{S, \Sigma} u_{0} d s \\
r\left(p, v_{0}\right)=-\int_{\Gamma_{S}} v_{0} \partial_{\mathbf{n}} V^{\Gamma_{S}, \Sigma} p d s+i \kappa \int_{S} \eta v_{0} V^{S, \Sigma} p d s \\
b(p, v)=\left\langle p, v_{1}-v_{0}\right\rangle_{\widetilde{H}^{-1 / 2}(\Sigma), H^{1 / 2}(\Sigma)}
\end{array}\right.
$$

and

$$
M=\widetilde{H}^{-1 / 2}(\Sigma) .
$$

For simplicity, an element $\left(v_{0}, v_{1}\right) \in X$ is denoted by a single symbol $v$.

\subsection{Well-posedness and stability estimates}

We first establish the coerciveness estimates and Brezzi's inf-sup condition that reduce problem (3.14) to a Fredholm alternative. Towards this end, we introduce the following notation:

$$
\left\{\begin{array}{l}
a^{(0)}(u, v)=\int_{\Omega_{S} \cap \Omega_{0}}\left(\nabla u_{0} \cdot \nabla v_{0}+u_{0} v_{0}\right) d x+\int_{\Omega_{1}} \chi\left(\nabla u_{1} \cdot \nabla v_{1}+u_{1} v_{1}\right) d x \\
d^{(0)}\left(u_{0}, v_{0}\right)=\left\langle\partial_{\mathbf{n}} N^{(0)} u_{0}, v_{0}\right\rangle_{H^{-1 / 2}\left(\Gamma_{S}\right), H^{1 / 2}\left(\Gamma_{S}\right)}
\end{array}\right.
$$

where $N^{(0)} u_{0}$ is the double-layer potentiel corresponding to the kernel of the Laplace equation and the curve $\Gamma_{\Sigma}$ created by the density $u_{0}$

$$
N^{(0)} u_{0}(x)=\int_{\Gamma_{\Sigma}} \frac{1}{2 \pi} \partial_{\mathbf{n}_{y}} \ln (|x-y|) u_{0}(y) d s_{y} .
$$

Let us consider the following variational problem

$$
\left\{\begin{array}{l}
(u, p) \in X \times M, \forall(v, q) \in X \times M \\
a^{(0)}(u, v)+d^{(0)}\left(u_{0}, v_{0}\right)+b(r, v)=\mathscr{L} v \\
b(q, u)=\langle q, \ell\rangle_{\widetilde{H}^{-1 / 2}(\Sigma), H^{1 / 2}(\Sigma)}
\end{array}\right.
$$

set with $\mathscr{L} \in X^{\prime}$, the space of continuous linear forms on $X$, and $\ell \in H^{1 / 2}(\Sigma)$. Clearly, usual properties of boundary integral operators readily yield that problems (3.14) and (3.17) differ from each other by compact bilinear forms only (cf., e.g., Buffa \& Hiptmair (2003); Hsiao \& Wendland (2008); Nédélec (2001)). For this poblem, we have the following well-posedness result. 
LEMMA 3.1 Problem (3.17) admits one and only one solution and there exists a constant $C$ independent of $\mathscr{L}$ and $\ell$ such that

$$
\|u\|_{X}+\|p\|_{\widetilde{H}^{-1 / 2}(\Sigma)} \leqslant C\left(\sup _{\|v\|_{X} \leqslant 1}|\mathscr{L} v|+\|\ell\|_{H^{1 / 2}(\Sigma)}\right) .
$$

Proof. Problem (3.17) is a saddle-point problem. Its well-posedness will result from the following Brezzi's conditions

$$
\begin{aligned}
& \exists \beta>0: \sup _{\|v\|_{X} \leqslant 1}|b(p, v)| \geqslant \beta\|p\|_{\widetilde{H}^{-1 / 2}(\Sigma)} \\
& \exists \gamma>0: a^{(0)}(u, \bar{u})+d^{(0)}\left(u_{0}, \overline{u_{0}}\right) \geqslant \gamma\|u\|_{X}^{2}
\end{aligned}
$$

for all $u \in V$ with

$$
V=\{v \in X ; b(q, v)=0, \forall q \in M\}
$$

(see for instance Brezzi \& Fortin (1991)). Since $b(p, v)=\left\langle p, v_{1}\right\rangle_{\widetilde{H}^{-1 / 2}(\Sigma), H^{1 / 2}(\Sigma)}$ for $v_{0}=0$, inf-sup condition (3.18) is immediately obtained by noticing that $\widetilde{H}^{-1 / 2}(\Sigma)$ is the dual space of $H^{1 / 2}(\Sigma)$ (cf., e.g., McLean (2000); see also (Steinbach, 2003, p. 79)). To prove (3.19), we first remember that $u=\left(u_{0}, u_{1}\right) \in V$ means that if $w$ is defined by $\left.w\right|_{\Omega_{0} \cap \Omega_{S}}=u_{0}$ and $\left.w\right|_{\Omega_{1}}=u_{1}$, then it is in $H^{1}\left(\Omega_{S}\right)$. Then, since $N^{(0)} u_{0}$ is a double-layer potential associated to the Laplace equation, it is an harmonic function in $\Omega_{0}$, i.e., $\Delta N^{(0)} u_{0}=0$ in $\Omega_{0}$, and satisfies $N^{(0)} u_{0} \in H^{1}\left(\Omega_{0} \cap B_{R}\right)$ for any ball $B_{R}$ centered at 0 and of radius $R$ (for this last property see, e.g., (McLean, 2000, p. 208)). Now, let $\varphi$ be a cut-off function in $\mathscr{D}\left(\mathbb{R}^{2}\right)$ equal to 1 on a ball $B_{R}$ with $R$ sufficiently large such that $\Omega_{0}^{+} \cup \Omega_{S} \subset B_{R}$. Using Green's formula, we can write

$$
\begin{aligned}
d^{(0)}\left(u_{0}, \overline{u_{0}}\right) & =\left\langle\partial_{\mathbf{n}} N^{(0)} u_{0}, \overline{\varphi u_{0}}\right\rangle_{H^{-1 / 2}\left(\Gamma_{S}\right), H^{1 / 2}\left(\Gamma_{S}\right)} \\
& =-\int_{\Omega_{0} \backslash \overline{\Omega_{S}}} \nabla N^{(0)} u_{0} \cdot \nabla \overline{\varphi u_{0}} d x \\
& =-\int_{\Omega_{0}} \nabla N^{(0)} u_{0} \cdot \nabla\left(\overline{\varphi u_{0}}\right) d x+\int_{\Omega_{0} \cap \Omega_{S}} \nabla N^{(0)} u_{0} \cdot \nabla \overline{u_{0}} d x
\end{aligned}
$$

since $\varphi$ is equal to 1 on $\Omega_{S}$. Using once more Green formula, we readily obtain

$$
\begin{aligned}
a^{(0)}(u, \bar{u})+d^{(0)}\left(u_{0}, \overline{u_{0}}\right) & =\int_{\Omega_{S}} \chi\left(|\nabla w|^{2}+|w|^{2}\right) d x \\
& +\int_{\Omega_{0} \cap \Omega_{S}} \nabla N^{(0)} u_{0} \cdot \nabla \overline{u_{0}} d x+\left\langle\partial_{\mathbf{n}} N^{(0)} u_{0}, \overline{u_{0}}\right\rangle_{H^{-1 / 2}\left(\Gamma_{\Sigma}\right), H^{-1 / 2}\left(\Gamma_{\Sigma}\right)} .
\end{aligned}
$$

Making use once more of the following properties of the double-layer potential $\Delta N^{(0)} u_{0}=0$ in $\Omega_{0}^{+} \cup \Omega_{0}$, $\left(\partial_{\mathbf{n}} N^{(0)} u_{0}\right)^{+}=\left(\partial_{\mathbf{n}} N^{(0)} u_{0}\right)^{-}=\partial_{\mathbf{n}} N^{(0)} u_{0}$ on $\Gamma_{\Sigma},\left(N^{(0)} u_{0}\right)^{+}-\left(N^{(0)} u_{0}\right)^{-}=\left.u_{0}\right|_{\Gamma_{\Sigma}}$, and $\left.\nabla N^{(0)} u_{0}\right|_{\Omega_{0}^{+}} \in$ $L^{2}\left(\Omega_{0}^{+}\right)$and $\left.\nabla N^{(0)} u_{0}\right|_{\Omega_{0}} \in L^{2}\left(\Omega_{0}\right)$, we can write

$$
\left\langle\partial_{\mathbf{n}} N^{(0)} u_{0}, \overline{u_{0}}\right\rangle_{H^{-1 / 2}\left(\Gamma_{\Sigma}\right), H^{-1 / 2}\left(\Gamma_{\Sigma}\right)}=\int_{\Omega_{0}^{+}}\left|\nabla N^{(0)} u_{0}\right|^{2} d x+\int_{\Omega_{0}}\left|\nabla N^{(0)} u_{0}\right|^{2} d x .
$$


10 of 24

We have denoted by a superscript + the trace on $\Gamma_{\Sigma}$ from the values of the corresponding function on $\Omega_{0}^{+}$and by a superscript - this trace from the values on $\Omega_{0}$. The end of the proof is obtained in a straightforward way by noticing that

$$
\begin{aligned}
\Re \int_{\Omega_{0} \cap \Omega_{S}} \nabla N^{(0)} u_{0} \cdot \nabla \overline{u_{0}} d x & \geqslant-\left\|\nabla N^{(0)} u_{0}\right\|_{L^{2}\left(\Omega_{0} \cap \Omega_{S}\right)}\left\|\nabla u_{0}\right\|_{L^{2}\left(\Omega_{0} \cap \Omega_{S}\right)} \\
& \geqslant-\frac{1}{2}\left(\left\|\nabla N^{(0)} u_{0}\right\|_{L^{2}\left(\Omega_{0} \cap \Omega_{S}\right)}^{2}+\left\|\nabla u_{0}\right\|_{L^{2}\left(\Omega_{0} \cap \Omega_{S}\right)}^{2}\right) .
\end{aligned}
$$

Keeping the above data and notation, we can now prove the following general stability result which clearly reduces to a Fredholm alternative (cf., e.g., Hsiao \& Wendland (2008); Taylor (1996)).

THEOREM 3.1 The variational problem

$$
\left\{\begin{array}{l}
(u, p) \in X \times M, \forall(v, q) \in X \times M \\
a(u, v)+d\left(u_{0}, v_{0}\right)+b(p, v)+r\left(u_{0}, v_{0}\right)=\mathscr{L} v \\
b(q, u)=\langle q, \ell\rangle_{\widetilde{H}^{-1 / 2}(\Sigma), H^{1 / 2}(\Sigma)}
\end{array}\right.
$$

admits one and only one solution satisfying

$$
\|w\|_{X}+\|r\|_{\widetilde{H}^{-1 / 2}(\Sigma)} \leqslant C\left(\sup _{\|v\|_{X} \leqslant 1}|\mathscr{L} v|+\|\ell\|_{H^{1 / 2}(\Sigma)}\right)
$$

with $C$ a constant independent of $\mathscr{L}$ and $\ell$.

Proof. We have seen right above that the well-posedness of problem (3.20) reduces to a Fredholm alternative. We thus focus on the uniqueness and consider $(u, p)$ a solution to (3.20) corresponding to $\mathscr{L}=0$ and $\ell=0$. At first, we note that, since $b(q, u)=0, \forall q \in \widetilde{H}^{-1 / 2}(\Sigma)$, we have $\left.u_{0}\right|_{\Sigma}=\left.u_{1}\right|_{\Sigma}$. Hence, if we define $w$ by $\left.w\right|_{\Omega_{0} \cap \Omega_{S}}=u_{0}$ and $\left.w\right|_{\Omega_{1}}=u_{1}$, we get that $w \in H^{1}\left(\Omega_{S}\right)$. The notation $w$ will stand also for the function defined on $\Gamma_{\Sigma}$ associated to $u_{0}$ and which coincides with $u_{0}$ on $\Gamma_{\Sigma}$ (see the definition of $X_{0}$ in (3.13)). To put problem (3.20) in a more concrete shape, let us introduce the following function $z$ expressed in terms of a single- and a double-layer potential, which as well-known yield a solution to the Helmholtz equation in $\Omega_{0}^{+} \cup \Omega_{0}$ outgoing at infinity

$$
z(x)=V^{*, \Sigma} p(x)-N^{*, \Gamma_{\Sigma}} w(x), \quad x \in \mathbb{R}^{2} \backslash \Gamma_{\Sigma} .
$$

Let us denote by $z^{ \pm}$and $\partial_{\mathbf{n}} z^{ \pm}$the traces of $z$ on $\Gamma_{\Sigma}$ where the signs \pm respectively indicate that the traces are taken from inside (resp. outside) the domain $\Omega_{0}^{+}$enclosed by $\Gamma_{\Sigma}$. Jump relations (cf., e.g., Colton $\&$ Kress (1983); Bendali \& Fares (2008))) connect these traces to that of $w$ on $\Sigma$ as

$$
z^{-}-z^{+}=w, \quad \partial_{\mathbf{n}} z^{-}-\partial_{\mathbf{n}} z^{+}=-p .
$$

Let now $S_{0}$ be the part of $\Gamma_{\Sigma}$ in $\overline{\Omega_{S}}$ (see Fig. 2). Observing that $\partial_{\mathbf{n}} z$ has no jump across $\Gamma_{\Sigma} \backslash \overline{S_{0}}$, by chosing $v \in X$ such that $v_{1}=0$ and $\operatorname{supp} v_{0}$ compact in $\Gamma_{\Sigma} \backslash \overline{S_{0}}$, we directly obtain that $\partial_{\mathbf{n}} z=0$ on $\Gamma_{\Sigma} \backslash \overline{S_{0}}$. In other words, both of $\partial_{\mathbf{n}} z^{+}$and $\partial_{\mathbf{n}} z^{-}$are in $\widetilde{H}^{-1 / 2}\left(S_{0}\right)$. As in the proof of lemma 3.17, since $z \in H^{1}\left(\Omega_{0} \cap B_{R}\right)$ for all $R$, and satisfies $\Delta z+\kappa^{2} z=0$ in $\Omega_{0} \cup \Omega_{0}^{+},\left.\partial_{\mathbf{n}} z\right|_{\Gamma_{S}}$ is well-defined in $H^{-1 / 2}\left(\Gamma_{S}\right)$ and belongs to $\widetilde{H}^{-1 / 2}(S)$. Going back to problem (3.20), we can thus see that $w, p$ and $z$ satisfy

$$
\begin{aligned}
& \int_{\Omega_{0}}\left(\nabla w \cdot \nabla v_{0}-\right.\left.\kappa^{2} w v_{0}\right) d x-i \kappa \int_{S} \eta w v_{0} d s+\int_{\Omega_{1}} \chi\left(\nabla w \cdot \nabla v_{1}-\kappa^{2} w v_{1}\right) d x \\
&\left\langle p, v_{1}-v_{0}\right\rangle_{\widetilde{H}^{-1 / 2}(\Sigma), H^{1 / 2}(\Sigma)}-\left\langle\partial_{\mathbf{n}} z, v_{0}\right\rangle_{\widetilde{H}^{-1 / 2}(S), H^{1 / 2}(S)}+i \kappa \int_{S} \eta z v_{0} d s=0
\end{aligned}
$$


for all $v_{0} \in H^{1}\left(\Omega_{0}\right)$ and $v_{1} \in H^{1}\left(\Omega_{1}\right)$. Green formula then directly gives that $\chi \partial_{\mathbf{n}} w$ has no jump across $\Sigma$, that $p=-\chi \partial_{\mathbf{n}} u$ and that $w$ satisfies

$$
\left\{\begin{array}{l}
\nabla \chi \nabla \cdot w+\chi \kappa^{2} n^{2} w=0 \text { in } \Omega_{S} \\
\chi \partial_{\mathbf{n}} w=0 \text { on } \Gamma \cap \partial \Omega_{S}
\end{array}\right.
$$

that is, $\chi \partial_{\mathbf{n}} w \in \widetilde{H}^{-1 / 2}(S)$. In the same way, we get also that

$$
\chi \partial_{\mathbf{n}} w-i \kappa \eta w=\partial_{\mathbf{n}} z-i \kappa \eta z \text { on } S
$$

The crucial point in the proof is then to take advantage of the fact that $\chi$ and $n$ are 1 in $\Omega_{0}$ for getting that

$\int_{\Omega_{0} \cap \Omega_{S}}\left(|\nabla(w-z)|^{2}-\kappa^{2}|w-z|^{2}\right) d x-i \kappa \int_{S} \eta|w-z|^{2} d s=-\left\langle\chi \partial_{\mathbf{n}} w-\partial_{\mathbf{n}} z^{-}, \overline{w-z^{-}}\right\rangle_{\widetilde{H}^{-1 / 2}\left(S_{0}\right), H^{1 / 2}\left(S_{0}\right)}$.

Then using jump relations (3.22) and the fact that both $\partial_{\mathbf{n}} z^{+}$and $\partial_{\mathbf{n}} z^{-}$are in $\widetilde{H}^{-1 / 2}\left(S_{0}\right)$, we can write

$$
\begin{aligned}
\int_{\Omega_{0} \cap \Omega_{S}}\left(|\nabla(w-z)|^{2}-\kappa^{2}|w-z|^{2}\right) d x-i \kappa \int_{S} \eta|w-z|^{2} d s & =-\left\langle\partial_{\mathbf{n}} z^{+}, \overline{z^{+}}\right\rangle_{\widetilde{H}^{-1 / 2}\left(S_{0}\right), H^{1 / 2}\left(S_{0}\right)} \\
& =-\left\langle\partial_{\mathbf{n}} z^{+}, \overline{z^{+}}\right\rangle_{H^{-1 / 2}\left(\Gamma_{\Sigma}\right), H^{1 / 2}\left(\Gamma_{\Sigma}\right)}
\end{aligned}
$$

As above, since $\Delta z+\kappa^{2} z=0$ in $\Omega_{0}^{+}$and $z \in H^{1}\left(\Omega_{0}^{+}\right)$, Green formula then gives that

$$
\int_{\Omega_{0} \cap \Omega_{S}}\left(|\nabla(w-z)|^{2}-\kappa^{2}|w-z|^{2}\right) d x-i \kappa \int_{S} \eta|w-z|^{2} d s+\int_{\Omega_{0}^{+}}\left(|\nabla z|^{2}-\kappa^{2}|z|^{2}\right) d x=0 .
$$

Taking the imaginary part of this equation, we obtain

$$
\int_{S} \eta|w-z|^{2} d s=0
$$

Therefore, we have that $\partial_{\mathbf{n}}(w-z)=w-z=0$ in a neighborhood of at least an interior point of $S$ since we can find at least one interior point of $S$ around which $\eta=1$. We then deduce that $w=z$ in $\Omega_{0} \cap \Omega_{S}$ by using, for instance, the Holmgren theorem (cf. Chazarain \& Piriou (1982)). It is then possible to extend $w$ by $z$ in all of $\Omega_{0}$. The end of the proof follows from the uniqueness of problem (2.1).

\section{Discretization and numerical solution}

After describing the coupling FEM-BEM approximation of problem (3.14), we establish its stability and convergence.

\subsection{The FEM-BEM approximation}

The domain $\Omega_{S}$ is meshed in triangles according to the general matching rules of conforming meshes of the FEM and in a way compatible with its decomposition in the two non-overlapping domains $\Omega_{1}$ and $\Omega_{0} \cap \Omega_{S}$. This induces a mesh in segments of $\Sigma$. The rest of $\Gamma_{\Sigma}$ is also meshed in segments. An instance of such a mesh is depicted in Fig. 4, which moreover exhibits the nodes used either for the FEM and the BEM. 


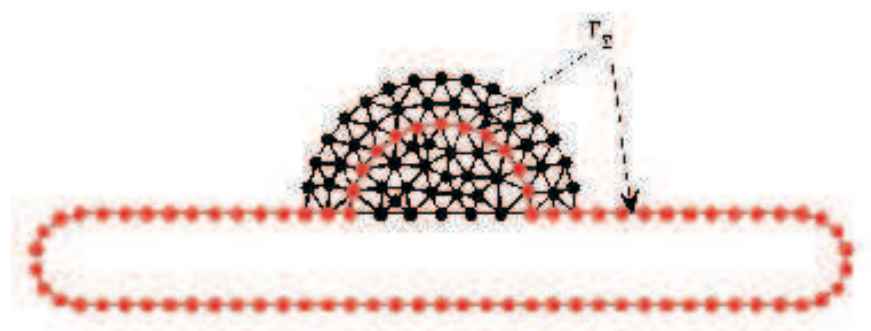

FIG. 4. Meshes and nodes of $\Omega_{S}$ and $\Gamma_{\Sigma}$ used for the FEM-BEM approximation.

Denoting by $h>0$ the usual mesh size of FEM approximations, we can then consider the FEM-BEM discrete version $X_{0}^{h}\left(\right.$ resp. $\left.X_{1}^{h}, M^{h}\right)$ of $X_{0}\left(\right.$ resp. $\left.X_{1}, M\right)$. A function $v^{h}$ in $X_{0}^{h}\left(\right.$ resp. $\left.X_{1}^{h}, M^{h}\right)$ is defined such that $v^{h}$ is globally continuous on $\overline{\Omega_{0} \cap \Omega_{S}} \cup \Gamma_{\Sigma}$ (resp. $\bar{\Omega}_{1}, \bar{\Sigma}$ ), and linear-affine on each triangle or segment included in this set. It is worth mentioning that the condition $M=\widetilde{H}^{-1 / 2}(\Sigma)$ is taken into account by compelling the nodal values of $p^{h} \in M^{h}$ to be zero at the end-points of $\Sigma$. The FEM-BEM space $X^{h}$ is associated with $X_{0}^{h}$ and $X_{1}^{h}$ in the same way as $X$ with $X_{0}$ and $X_{1}$ except that, in order to avoid the usual difficulties raised by the cross-points (cf., e.g., Bendali \& Boubendir (2006)), $v_{0}^{h} \in X_{0}^{h}$ and $v_{1}^{h} \in X_{1}^{h}$ are assumed to share the same nodal value at the end-points of $\Sigma$. The norm on $X^{h}$ is that on $X$, that is,

$$
\left\|v^{h}\right\|_{X}=\sqrt{\left\|\left.v_{0}^{h}\right|_{\Omega_{0}}\right\|_{H^{1}\left(\Omega_{0}\right)}^{2}+\left\|v_{1}^{h}\right\|_{H^{1}\left(\Omega_{1}\right)}^{2}+\left\|\left.v_{0}^{h}\right|_{\Gamma_{\Sigma}}\right\|_{H^{1 / 2}\left(\Gamma_{\Sigma}\right)}^{2}} .
$$

\subsection{Stability and convergence of the FEM-BEM scheme}

The stability and the convergence of the above FEM-BEM scheme are embodied in the following theorem. In the sequel, $C$ stands for various constants, not the same in all instances, always independent of $h$. We also neglect the consistency error coming from the approximation of curved interfaces and boundaries by straight lines (see how such an error can be taken into account in Bendali \& Souilah (1994) for instance).

THEOREM 4.1 Assume that the mesh of $\Sigma$ is globally quasi-uniform in the meaning

$$
h_{\max }^{\Sigma} / h_{\min }^{\Sigma} \leqslant C
$$

where $h_{\max }^{\Sigma}$ and $h_{\min }^{\Sigma}$ are respectively the lengths of the largest and the smallest segment of $\Sigma$. There exists $h_{0}>0$ and a constant $C$ independent of $0<h \leqslant h_{0}$ such that, if $\mathscr{L}_{h}$ and $\ell_{h}$ are linear forms on respectively $X^{h}$ and $M^{h}$, the problem

$$
\left\{\begin{array}{l}
\left(w^{h}, z^{h}\right) \in X^{h} \times M^{h}, \forall\left(v^{h}, q^{h}\right) \in X^{h} \times M^{h}, \\
a\left(w^{h}, v^{h}\right)+d\left(w_{0}^{h}, v_{0}^{h}\right)+b\left(z^{h}, v^{h}\right)+r\left(z^{h}, v_{0}\right)=\mathscr{L}_{h} v^{h}, \\
b\left(q^{h}, w^{h}\right)=\ell_{h} q^{h},
\end{array}\right.
$$


admits one and only one solution such that

$$
\left\|w^{h}\right\|_{X}+\left\|z^{h}\right\|_{\widetilde{H}^{-1 / 2}(\Sigma)} \leqslant C\left(\sup _{\left\|v^{h}\right\|_{X} \leqslant 1}\left|\mathscr{L}_{h} v_{h}\right|+\sup _{\left\|q^{h}\right\|_{\tilde{H}^{-1 / 2}(\Sigma)} \leqslant 1}\left|\ell_{h} q^{h}\right|\right) .
$$

Proof. The proof is obtained from the coerciveness properties of the bilinear form $\left(w^{h}, v^{h}\right) \rightarrow a\left(w^{h}, v^{h}\right)+$ $d\left(w_{0}^{h}, v_{0}^{h}\right)$, the usual stability estimates for the Galerkin approximation of the problems whose wellposedness is established from a Fredholm alternative, (see, for instance, Chen \& Zhou (1992)) and the following Brezzi condition for saddle-point problems

$$
\exists \gamma>0 \text { independent of } h: \sup _{\left\|v^{h}\right\|_{X} \leqslant 1}\left|b\left(p^{h}, v^{h}\right)\right| \geqslant \gamma\left\|p^{h}\right\|_{\widetilde{H}^{-1 / 2}(\Sigma)}, \forall p^{h} \in M^{h} .
$$

All these properties can be established in a straightforward way except the above stability condition which is an immediate consequence of the following one

$$
\exists \alpha>0 \text { independent of } h: \sup _{\left\|v_{1}^{h}\right\|_{H^{1}\left(\Omega_{1}\right)} \leqslant 1}\left|\left\langle p^{h}, v^{h}\right\rangle_{\widetilde{H}^{-1 / 2}(\Sigma), H^{1 / 2}(\Sigma)}\right| \geqslant \alpha\left\|p^{h}\right\|_{\widetilde{H}^{-1 / 2}(\Sigma)}, \forall p^{h} \in M^{h} .
$$

Fortin's lemma (cf. (Brezzi \& Fortin, 1991, p. 58)) directly yields this estimate from the following property: the $L^{2}$ projection onto $V^{h}$, the space of functions continuous on $\Sigma$ and linear-affine on each segment, is bounded uniformly in $h$ as a map from $H^{1 / 2}(\Sigma)$ into itself (cf., e.g., (Steinbach, 2003, p. 21)).

The following corollary, proving the convergence of the FEM-BEM approximation of the solution to (2.1), is an immediate consequence of the above theorem.

COROLLARY 4.1 Under the general conditions of theorem 4.1, there exists $h_{0}>0$ such that, for $0<$ $h \leqslant h_{0}$, the following estimate holds true

$$
\left\|u^{h}-u\right\|_{X}+\left\|p^{h}-p\right\|_{\widetilde{H}^{-1 / 2}(\Sigma)} \leqslant C\left(\inf _{v^{h} \in X^{h}}\left\|v^{h}-u\right\|_{X}+\inf _{q^{h} \in M^{h}}\left\|q^{h}-p\right\|_{\widetilde{H}^{-1 / 2}(\Sigma)}\right)
$$

where $(u, p)$ is the solution to the continuous problem (3.14), and $\left(u^{h}, p^{h}\right)$ is the solution of the following discrete one

$$
\left\{\begin{array}{l}
\left(u^{h}, p^{h}\right) \in X^{h} \times M^{h}, \forall\left(v^{h}, q^{h}\right) \in X^{h} \times M^{h} \\
a\left(u^{h}, v^{h}\right)+d\left(u_{0}^{h}, v_{0}^{h}\right)+b\left(p^{h}, v^{h}\right)+r\left(p^{h}, v_{0}^{h}\right)=\left\langle f, v_{1}^{h}\right\rangle_{\widetilde{H}^{-1 / 2}\left(\Gamma_{\text {slot }}\right) H^{1 / 2}\left(\Gamma_{\text {slot }}\right)}, \\
b\left(q^{h}, u^{h}\right)=0
\end{array}\right.
$$

\subsection{Numerical implementation}

The general strategy for solving the discrete problem (4.5) is to use an iterative procedure to uncouple the FEM and the BEM solutions in order to deal with linear systems with either a sparse or a dense matrix. Let us first describe the procedure as a successive approximations method. The iterative process is actually accelerated by means of a suitable Krylov technique. For the sake of clarity, we use the following projector $v^{h} \rightarrow \Pi v_{0}^{h}$ obtained by setting to zero each nodal value of $v_{0}^{h}$ not on $\overline{\Omega_{S}}$. In matrix form, $\Pi$ just corresponds to a partitioning technique. The superscript $h$ is removed to simplify the notation. 
14 of 24

- Start with $u^{(0)}=0$ and $p^{(0)}=0$.

- For $m=0,1, \ldots$ until convergence solve

$$
\left\{\begin{array}{l}
\quad \begin{array}{l}
\left.u^{(m+1)}, p^{(m+1)}\right) \in X^{h} \times M^{h}, \forall(v, q) \in X^{h} \times M^{h}, \\
a\left(u^{(m+1)}, v\right)+d\left((1-\Pi) u_{0}^{(m+1)}, v_{0}\right)+b\left(p^{(m+1)}, v\right)= \\
b\left(q, u^{(m+1)}\right)=0, \\
\left\langle f, v_{1}^{h}\right\rangle_{\widetilde{H}^{-1 / 2}\left(\Gamma_{\text {slot }}\right), H^{1 / 2}\left(\Gamma_{\text {slot }}\right)}-d\left(\Pi u_{0}^{(m)}, v_{0}\right)-r\left(p^{(m)}, v_{0}\right)
\end{array}
\end{array}\right.
$$

Actually, since $a_{0}\left(u_{0},(1-\Pi) v_{0}\right)=b_{0}\left(p,(1-\Pi) v_{0}\right)=0$, the problem (4.6) consists of two uncoupled problems

$$
\left\{\begin{array}{c}
a_{1}\left(u_{1}^{(m+1)}, v_{1}\right)+a_{0}\left(\Pi u_{0}^{(m+1)}, \Pi v_{0}\right)+b_{1}\left(p^{(m+1)}, v_{1}\right)-b_{0}\left(p^{(m+1)}, \Pi v_{0}\right)= \\
\left\langle f, v_{1}^{h}\right\rangle_{\widetilde{H}^{-1 / 2}\left(\Gamma_{\text {slot }}\right) H^{1 / 2}\left(\Gamma_{\text {slot }}\right)}-d\left(\Pi u_{0}^{(m)}, \Pi v_{0}\right)-r\left(p^{(m)}, \Pi v_{0}\right) \\
b_{1}\left(q, u_{1}^{(m+1)}\right)-b_{0}\left(q, \Pi u_{0}^{(m+1)}\right)=0, \\
d\left((1-\Pi) u_{0}^{(m+1)},(1-\Pi) v_{0}\right)=-d\left(\Pi u_{0}^{(m)},(1-\Pi) v_{0}\right)-r\left(p^{(m)},(1-\Pi) v_{0}\right) .
\end{array}\right.
$$

Moreover, problem (4.7) can be solved in two steps. The first one is a simple FEM solution on $\Omega_{S}$ that determines the nodal values of $u$ there. This problem is obtained by compelling the test function $v$ to satisfy the constraint, that is, to be continuous also at the nodes in the interior of $\Sigma$

$$
\left\{\begin{array}{l}
\widetilde{u}^{(m+1)} \in V^{h}, \forall v \in V^{h} \\
\int_{\Omega_{S}} \chi\left(\nabla \widetilde{u}^{(m+1)} \cdot \nabla v-\kappa^{2} n^{2} \widetilde{u}^{(m+1)} v\right) d x-i \kappa \int_{S} \eta \widetilde{u}^{(m+1)} v d s= \\
\left\langle f, v_{1}^{h}\right\rangle_{\widetilde{H}^{-1 / 2}\left(\Gamma_{\text {slot }}\right), H^{1 / 2}\left(\Gamma_{\text {slot }}\right)}-d\left(\Pi u_{0}^{(m)}, \Pi v_{0}\right)-r\left(p^{(m)}, \Pi v_{0}\right)
\end{array}\right.
$$

with

$$
V^{h}=\left\{v^{h} \in \mathscr{C}^{0}\left(\bar{\Omega}_{S}\right) ; v^{h} \text { linear-affine on each triangle }\right\}
$$

and $\widetilde{u}^{(m+1)}$ is such that $\widetilde{u}^{(m+1)}\left|\Omega_{0} \cap \Omega_{S}=u_{0}^{(m+1)}\right| \Omega_{0} \cap \Omega_{S}$ and $\widetilde{u}^{(m+1)} \mid \Omega_{1}=u_{1}^{(m+1)}$. The multiplier $p^{(m+1)}$ is next obtained by solving the variational system whose underlying linear system is simply a boundary mass matrix associated with the mesh on $\Sigma$

$$
b_{1}\left(p^{(m+1)}, v_{1}\right)=-a_{1}\left(u_{1}^{(m+1)}, v_{1}\right), \forall v_{1} \in M^{h} .
$$

In addition to designing a procedure which makes it possible to solve separately sparse and dense matrices, we also get the following theorem.

THEOREM 4.2 The above iterative algorithm does not break down.

Proof. It is sufficient to prove that the above three problems are well-posed. Problem (4.9) corresponds to a FE approximation of a boundary-value problem set in terms of a Helmholtz equation with variable coefficients and a strictly absorbing impedance condition in a part of the boundary. In this way, it satisfies strong coerciveness properties which yields that the related linear system can be solved in a stable way. Problem (4.10) is a linear system only posed in terms of a mass matrix. Problem (4.8) corresponds to an integral equation set on an open curve and solved by a BEM, whose well-posedness is well-known (cf., e.g., Hsiao \& Wendland (2008); Maischak et al. (1997)).

We will refer below to this approach as the LRC (Localized Radiation Condition) formulation. 


\section{Alternative approaches}

In this section, we consider alternative approaches for solving problem (2.1). The goal is to validate the LRC formulation by first testing its accuracy through its comparison with standard methods. Next we compare its efficiency with methods based on other domain decomposition principles.

\subsection{Direct methods}

"Direct methods" means here that the underlying linear system can be solved by usual Gaussian eliminations.

5.1.1 Boundary elements formulations. When $\chi$ and $n$ are constant, the problem can be addressed using only a BEM. We have chosen the reduction to a system of first kind Boundary Integral Equations (BIE), as is done in von Petersdorff (1989), but with a more convenient way in expressing the constraints on the unknowns of the BIE. When internal resonances do not affect the well-posedness of such kinds of formulations, they are known to deliver very accurate results. The formulation of this integral equation makes use of the following space

$$
\begin{aligned}
\mathbb{X}^{1 / 2}=\left\{\left(v_{0}, v_{1}\right)\right. & \in H^{1 / 2}\left(\partial \Omega_{0}\right) \times H^{1 / 2}\left(\partial \Omega_{1}\right) ; \\
\exists v & \left.\in H_{\mathrm{loc}}^{1}\left(\mathbb{R}^{2}\right), v_{0}=\left.v\right|_{\partial \Omega_{0}}, v_{1}=\left.v\right|_{\partial \Omega_{1}}\right\}
\end{aligned}
$$

defined on the skeleton made of the boundaries and the interfaces involved in the problem. Conveniently, we use the notation $\partial \Omega_{0}$ to refer to the boundary of $\Omega_{0}$. Clearly from (2.4), the traces of the solution $u$ to $(2.1)$ are in $\mathbb{X}^{1 / 2}$. In the same way, we denote the flux variables associated with $u$ as follows

$$
p_{0}=\partial_{\mathbf{n}_{0}} u_{0}, \quad p_{1}=\chi \partial_{\mathbf{n}_{1}} u_{1}
$$

where $\mathbf{n}_{i}(i=0,1)$ stands for the unit normal to $\partial \Omega_{i}$ directed outwards $\Omega_{i}$. The Green formula directly yields that $\left(p_{0}, p_{1}\right)$ satisfies

$$
\left\langle p_{0}, v_{0}\right\rangle_{0}+\left\langle p_{1}, v_{1}\right\rangle_{1}=\left\langle f, v_{1}\right\rangle_{1}
$$

for all $\left(v_{0}, v_{1}\right) \in \mathbb{X}^{1 / 2}$. For simplicity, we have denoted by subscript 0 or 1 , the duality brackets between $H^{-1 / 2}\left(\partial \Omega_{0}\right)$ and $H^{1 / 2}\left(\partial \Omega_{0}\right)$, and between $H^{-1 / 2}\left(\partial \Omega_{1}\right)$ and $H^{1 / 2}\left(\partial \Omega_{1}\right)$ respectively. Clearly this condition defines a closed linear-affine subspace $\mathbb{X}^{-1 / 2}(f)$ of $H^{-1 / 2}\left(\partial \Omega_{0}\right) \times H^{-1 / 2}\left(\partial \Omega_{1}\right)$ whose underlying subspace is

$$
\begin{aligned}
& \mathbb{X}^{-1 / 2}=\left\{\left(q_{0}, q_{1}\right) \in H^{-1 / 2}\left(\partial \Omega_{0}\right) \times H^{-1 / 2}\left(\partial \Omega_{1}\right) ;\right. \\
&\left.\left\langle q_{0}, v_{0}\right\rangle_{0}+\left\langle q_{1}, v_{1}\right\rangle_{1}=0, \forall\left(v_{0}, v_{1}\right) \in \mathbb{X}^{1 / 2}\right\}
\end{aligned}
$$

Actually condition (5.2) expresses that

$$
\left.p_{0}\right|_{\Sigma}+\left.p_{1}\right|_{\Sigma}=0,\left.\quad p_{0}\right|_{\Gamma \cap \partial \Omega_{0}}=0,\left.\quad p_{1}\right|_{\Gamma \cap \partial \Omega_{1}}=f
$$

and $\left(q_{0}, q_{1}\right) \in \mathbb{X}^{-1 / 2}$ that $\left(q_{0}, q_{1}\right)$ satisfies (5.4) with $f=0$. The matching on the traces of $u$ can also be expressed in a dual way as

$$
\begin{aligned}
& \mathbb{X}^{1 / 2}=\left\{\left(v_{0}, v_{1}\right) \in H^{1 / 2}\left(\partial \Omega_{0}\right) \times H^{1 / 2}\left(\partial \Omega_{1}\right)\right. \\
& \quad\left\langle q_{0}, v_{0}\right\rangle_{H^{-1 / 2}}\left(\partial \Omega_{0}\right), H^{1 / 2}\left(\partial \Omega_{0}\right) \\
&
\end{aligned}
$$


16 of 24

Using the integral representations of $u_{0}$ in $\Omega_{0}$ in terms of $p_{0}$ and $\left.u_{0}\right|_{\partial \Omega_{0}}$, and that of $u_{1}$ in $\Omega_{1}$ from $p_{1}$ and $u_{1} \mid \partial \Omega_{1}$

$$
u_{0}(x)=V_{0} p_{0}(x)+N_{0} u_{0}(x)\left(x \in \Omega_{0}\right), \quad u_{1}=\chi^{-1} V_{1} p_{1}+N_{1} u_{1}\left(x \in \Omega_{1}\right) .
$$

Here, $V_{0}$ and $V_{1}$ are the single-layer potentials for respectively the wave numbers $\kappa$ and $\kappa_{1}=\kappa n$, and $N_{0}, N_{1}$ are the double-layer potentials defined using $\mathbf{n}_{0}, \kappa$ and $\mathbf{n}_{1}, \kappa_{1}$ respectively. We can readily solve the BIEs system as

$$
\left\{\begin{array}{l}
\left(u_{0}, u_{1}\right) \in \mathbb{X}^{1 / 2},\left(p_{0}, p_{1}\right) \in \mathbb{X}^{-1 / 2}(f), \forall\left(v_{0}, v_{1}\right) \in \mathbb{X}^{1 / 2}, \forall\left(q_{0}, q_{1}\right) \in \mathbb{X}^{-1 / 2} \\
\left\langle\partial_{\mathbf{n}_{0}} u_{0}, v_{0}\right\rangle_{0}-\left\langle q_{0}, u_{0}\right\rangle_{0}+\left\langle\chi \partial_{\mathbf{n}_{1}} u_{1}, v_{1}\right\rangle_{1}-\left\langle q_{1}, u_{1}\right\rangle_{1}=\left\langle f, v_{1}\right\rangle_{1} .
\end{array}\right.
$$

From usual trace formulas of single- and double-layer potentials (cf., e.g., Hsiao \& Wendland (2008); Bendali \& Fares (2008); Colton \& Kress (1983)), this system can be written in the explicit form

$$
\left\{\begin{array}{l}
\left(u_{0}, u_{1}\right) \in \mathbb{X}^{1 / 2},\left(p_{0}, p_{1}\right) \in \mathbb{X}^{-1 / 2}(f), \forall\left(v_{0}, v_{1}\right) \in \mathbb{X}^{1 / 2}, \forall\left(q_{0}, q_{1}\right) \in \mathbb{X}^{-1 / 2} \\
\left\langle\partial_{\mathbf{n}_{0}} N_{0} u_{0}, v_{0}\right\rangle_{0}+\left\langle\chi \partial_{\mathbf{n}_{1}} N_{1} u_{1}, v_{1}\right\rangle_{1}-\left\langle q_{0}, N_{0} u_{0}\right\rangle_{0}-\left\langle q_{1}, N_{1} u_{1}\right\rangle_{1} \\
\quad-\left\langle p_{0}, N_{0} v_{0}\right\rangle_{0}-\left\langle p_{1}, N_{1} v_{1}\right\rangle_{1}-\left\langle V_{0} p_{0}, q_{0}\right\rangle_{0}-\left\langle\chi^{-1} V_{1} p_{1}, q_{1}\right\rangle_{1}=\frac{1}{2}\left\langle f, v_{1}\right\rangle_{1} .
\end{array}\right.
$$

The discretization of this system by a BEM involving only globally continuous linear-affine functions on each segment of the above skeleton is straightforward and is not recalled here (cf., e.g., Chen \& Zhou (1992); Bendali \& Fares (2008); von Petersdorff (1989)).

5.1.2 FEM-BEM coupling. When $\chi$ or $n$ vary in $\Omega_{1}$, formulation (5.5) does not apply since the explicit representation of $u_{1}$ in terms of the Cauchy data $\left.u_{1}\right|_{\partial \Omega_{1}}$ and $p_{1}=\left.\chi \partial_{\mathbf{n}_{1}} u_{1}\right|_{\partial \Omega_{1}}$ is no more available. However to obtain a FEM-BEM coupling, we keep the variational system (5.5) in which $u_{1}$ represents the first trace of $u_{1}$ on $\partial \Omega_{1}$ and express $\chi \partial_{\mathbf{n}_{1}} u_{1} \mid \partial \Omega_{1}$ in terms of a Green formula

$$
\left\{\begin{aligned}
\left(u_{0}, u_{1}\right) \in \mathbb{X}, & \left(p_{0}, p_{1}\right) \in \mathbb{X}^{-1 / 2}(f), \forall\left(v_{0}, v_{1}\right) \in \mathbb{X}, \forall\left(q_{0}, q_{1}\right) \in \mathbb{X}^{-1 / 2} \\
\left\langle\partial_{\mathbf{n}_{0}} N_{0} u_{0}, v_{0}\right\rangle_{0} & -\left\langle q_{0}, N_{0} u_{0}\right\rangle_{0}-\left\langle p_{0}, N_{0} v_{0}\right\rangle_{0}-\left\langle V_{0} p_{0}, q_{0}\right\rangle_{0}+\frac{1}{2}\left\langle p_{0}, v_{0}\right\rangle_{0} \\
& +\int_{\Omega_{1}} \chi\left(\nabla u_{1} \cdot \nabla v_{1}-\kappa^{2} n^{2} u_{1} v_{1}\right) d x-\frac{1}{2}\left\langle q_{1}, u_{1}\right\rangle_{1}=\left\langle f, v_{1}\right\rangle_{1},
\end{aligned}\right.
$$

where

$$
\mathbb{X}=\left\{\left(u_{0}, u_{1}\right) \in H^{1 / 2}\left(\partial \Omega_{0}\right) \times H^{1}\left(\Omega_{1}\right) ;\left(u_{0}, u_{1} \mid \partial \Omega_{1}\right) \in \mathbb{X}^{1 / 2}\right\} .
$$

To solve this system, we mesh $\Omega_{1}$ in triangles and $\partial \Omega_{0}$ in segments, as was used in the case of the LRC formulation. We also use the same type of approximation, for trial and test function, as for the FEM-BEM formulation. Clearly the linear system associated with this formulation, referred to as the FEBE formulation in section 5.3, has a matrix which is partly sparse and partly dense. It can thus lead to specific difficulties when dealing with the huge size linear systems which is the inherent characteristic to such a kind of problems in 3D.

However, to compare these kinds of formulations with the above LRC formulation on the same basis, we will also consider an adaptation of this FEM-BEM coupling by setting the problem with varying coefficients in $\Omega_{S}$ after extending $\chi$ and $n$ in the homogeneous part of this domain by 1 . The resulting method will be referred to as the FEBE2 formulation. 


\subsection{Iterative methods}

We develop here some iterative methods, based on a suitable use of DDM techniques, that avoid solving linear systems whose matrix is partly sparse and partly dense.

5.2.1 A primal domain decomposition method. The domain decomposition method used here is based on the non-overlapping partition of of $\Omega$ into $\Omega_{0}$ and $\Omega_{1}$. The primal DDMs consists of operating the Dirichlet-to-Neumann map $T_{i}$ for $\Omega_{i}(i=0,1)$ (cf., e.g., Gosselet \& Rey (2006)). These maps are defined as follows

$$
\begin{aligned}
& \left\{\begin{array}{l}
w_{0} \in H_{\text {loc }}^{1}\left(\bar{\Omega}_{0}\right), \\
\Delta w_{0}+\kappa^{2} w_{0}=0 \text { in } \Omega_{0}, \\
w_{0}=\varphi_{0} \text { on } \partial \Omega_{0}, \\
\lim _{|x| \rightarrow \infty}|x|^{1 / 2}\left(\partial_{|x|} w_{0}-i \kappa w_{0}\right)=0,
\end{array}\right. \\
& \left\{\begin{array}{l}
w_{1} \in H^{1}\left(\Omega_{1}\right), \\
\nabla \cdot \chi \nabla w_{1}+\chi \kappa^{2} n^{2} w_{1}=0 \text { in } \Omega_{1}, \\
w_{1}=\varphi_{1} \text { on } \partial \Omega_{1},
\end{array}\right. \\
& T_{0} \varphi_{0}=\partial_{\mathbf{n}_{0}} w_{0}\left|\partial \Omega_{0} \in H^{-1 / 2}\left(\partial \Omega_{0}\right), \quad T_{1} \varphi_{1}=\chi \partial_{\mathbf{n}_{1}} w_{1}\right| \partial \Omega_{1} \in H^{-1 / 2}\left(\partial \Omega_{1}\right) .
\end{aligned}
$$

for $\varphi_{i}$ given in $H^{1 / 2}\left(\partial \Omega_{i}\right)$, and assuming that the corresponding Dirichlet problem for the Helmholtz equation is well-posed. The equations, corresponding to the continuous problem, can then be set using the above framework

$$
\left\{\begin{array}{l}
\left(u_{0}, u_{1}\right) \in \mathbb{X}^{1 / 2}, \forall\left(v_{0}, v_{1}\right) \in \mathbb{X}^{1 / 2} \\
\left\langle T_{0} u_{0}, v_{0}\right\rangle_{0}+\left\langle T_{1} u_{1}, v_{1}\right\rangle_{1}=\left\langle f, v_{1}\right\rangle_{1} .
\end{array}\right.
$$

Obviously, the equations of this system are set in an implicit way only.

The discretization of the coupling FEM-BEM system is based on the following approximation of the previous Dirichlet-to-Neumann maps. The approximation $T_{0}^{h}$ of $T_{0}$ is carried out by means of the following BEM discretization of the Burton-Miller BIE (cf., e.g., Bendali \& Fares (2008))

$$
\left\{\begin{array}{l}
\varphi_{0} \text { given in }\left.X_{0}^{h}\right|_{\partial \Omega_{0}}, p_{0}=\left.T_{0}^{h} \varphi_{0} \in X_{0}^{h}\right|_{\partial \Omega_{0}},\left.\forall v_{0} \in X_{0}^{h}\right|_{\partial \Omega_{0}}, \\
\left\langle\left(\frac{1}{2}-i \kappa V_{0}\right) p_{0}, v_{0}\right\rangle_{0}+\left\langle p_{0}, N_{0} v_{0}\right\rangle_{0}=\left\langle\left(\partial_{\mathbf{n}_{0}} N_{0}-i \kappa\left(\frac{1}{2}-N_{0}\right)\right) \varphi_{0}, v_{0}\right\rangle_{0},
\end{array}\right.
$$

where $\left.X_{0}^{h}\right|_{\partial \Omega_{0}}$ is the space generated by the restriction to $\partial \Omega_{0}$ of the functions $v_{0}^{h}$ belonging to the space $X_{0}^{h}$ previously defined in the context of the LRC formulation.

The approximation $T_{1}^{h}$ of $T_{1}$ is obtained by a standard FEM static condensation performed as follows. Let $K$ be the sparse matrix defined by the following identification

$$
\left\{\begin{array}{l}
u_{1} \in X_{1}^{h}, v_{1} \in X_{1}^{h} \\
\int_{\Omega_{1}} \chi\left(\nabla u_{1} \cdot \nabla v_{1}-\kappa^{2} n^{2} u_{1} v_{1}\right) d x=\left[v_{1}\right]^{\top} K\left[u_{1}\right]
\end{array}\right.
$$

where $X_{1}^{h}$ is the FEM space introduced above for the LRC formulation, and $\left[u_{1}\right]$ is the column-vector whose components are the corresponding nodal values of $u_{1}$. Let $I$ and $B$ be the sets of indices corresponding to the interior and boundary nodal values respectively. Partitioning

$$
K=\left[\begin{array}{ll}
K_{I I} & K_{I B} \\
K_{B I} & K_{B B}
\end{array}\right]
$$


accordingly, we get the nodal values $\left[p_{1}\right]$ of $p_{1}=T_{1}^{h} \varphi_{1}$ from those $\left[\varphi_{1}\right]$ of $\varphi_{1}$ in two sweeps:

- solve the linear system (corresponding to a Dirichlet problem with $\left[u_{1}\right]_{B}=\left[\varphi_{1}\right]$ )

$$
K_{I I}\left[u_{1}\right]_{I}=-K_{I B}\left[u_{1}\right]_{B}
$$

- form $\left[p_{1}\right]$

$$
\left[p_{1}\right]=K_{B I}\left[u_{1}\right]_{I}
$$

Denoting by

$$
\mathbb{X}_{\Sigma}^{h}=\left\{\left.\left(v_{0}, v_{1}\right) \in X_{0}^{h}\right|_{\partial \Omega_{0}} \times X_{1}^{h} ;\left.v_{0}\right|_{\Sigma}=\left.v_{1}\right|_{\Sigma}\right\},
$$

we obtain the discrete system related to the primal DDM

$$
\left\{\begin{array}{l}
\left(u_{0}, u_{1}\right) \in \mathbb{X}_{\Sigma}^{h}, \forall\left(v_{0}, v_{1}\right) \in \mathbb{X}_{\Sigma}^{h}, \\
\left\langle T_{0}^{h} u_{0}, v_{0}\right\rangle_{0}+\left\langle T_{1}^{h} u_{1}, v_{1}\right\rangle_{1}=\left\langle f, v_{1}\right\rangle_{1} .
\end{array}\right.
$$

Effectively, it is not possible to formulate the equations corresponding to problem (5.17) as a linear system. However, it is possible to perform a matrix-vector product using the expression (5.15) and the solution of (5.12). As a result, the system (5.17) can only be solved using a Krylov method. We will refer to this procedure as the P-DDM approach.

The main flaw of the above DDM is that the solution of the linear system (5.14) may be unsafe or can even break down if $\kappa$ corresponds to an internal resonance in $\Omega_{1}$. The expression of the matching conditions initiated by Deprés Després (1993) is generally used to cure this drawback for this kind of wave propagation problems. Such an approach is generally referred to as mixed DDM in the literature on domain decomposition methods (cf., e.g., Gosselet \& Rey (2006)). We have adapted this method with a special handling of cross-points as in Bendali \& Boubendir (2006). However, because of the type of geometry treated in this paper, several numerical experiments revealed that this method is the most expensive and the slowest in converging. For these reasons, we do not consider it as an alternative approach for the problem we are solving.

\subsection{Numerical experiments}

We end this section by some numerical experiments validating the LRC formulation. They consist of demonstrating its efficiency over the alternative techniques developed in section 5 .

5.3.1 Description of the test-case. The geometry of the test-case considered here is depicted in Fig. 5. It depends on a parameter $L$ used to set a large size for the impenetrable domain relatively to the zone meshed in triangles as shown in Fig. 5. By varying this parameter, we test each numerical technique in terms of accuracy, CPU time, and convergence for the iterative ones. The lengths are expressed in wavelength units. To be able to compare the LRC formulation with the BE one, we suppose $\chi$ and $n$ constant in $\Omega_{1}$. More precisely, we choose $\chi=1 / 4$ and $n=2(1+i)$, which correspond to a magnetic material in electromagnetism. The sources are located on the segment $\left\{x_{2}=0,-0.25<x_{1}<0.25\right\}$ and are given by the Gaussian function $f\left(x_{1}\right)=-\exp \left(-\left(10 x_{1}\right)^{2}\right)$.

The mesh used is of 20 points by wavelength in the free propagation zone and 15 points by wavelength in the material for the FEM-BEM formulations. The BE formulation is meshed using 20 points by wavelength for both the free propagation zone and the material. Since it is well-established that the $\mathrm{BE}$ approach is much less sensitive to the dispersion error than the FEM formulation, we use it here as the reference solution to determine the accuracy for the other methods. 


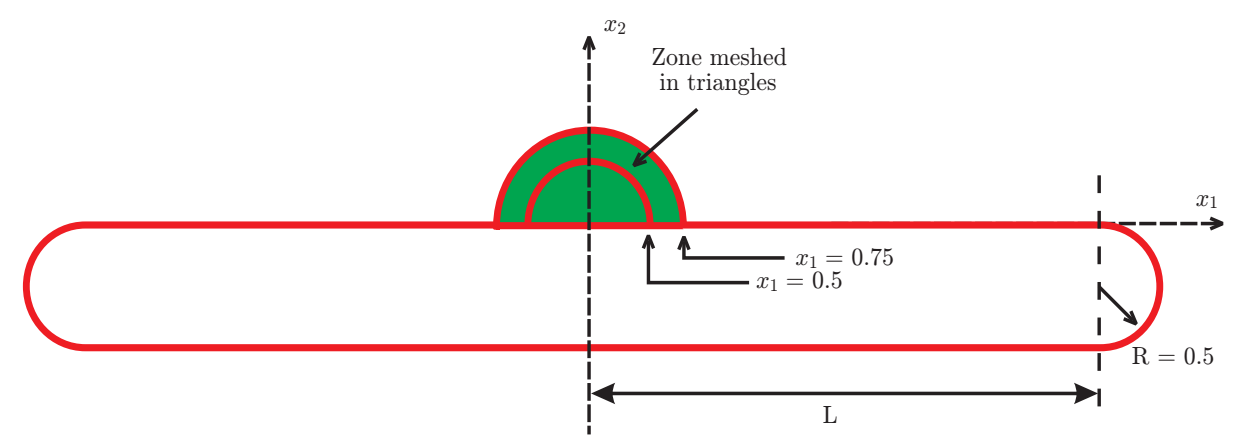

FIG. 5. Geometry of the test-case

5.3.2 Numerical experiments. The accuracy of each formulation is measured by comparing the far field pattern with the one obtained using the BE approach. The far field is expressed in $\mathrm{dB}$ as follows

$$
s(\theta)=10 \log _{10}\left(2 \pi\left|a_{0}(\theta)\right|^{2}\right)
$$

with

$$
u(x)=\frac{e^{i \kappa r}}{\sqrt{r}} a_{0}(\theta)+o\left(\frac{1}{\sqrt{ } r}\right),
$$

$(r, \theta)$ being the polar coordinates of the point $x \in \mathbb{R}^{2}$.

All the iterative methods are solved using the GMRES algorithm (cf., e.g. Saad (1996)). Since this procedure does not seem to be well-known, we sketch its broad outlines. Assume that a linear problem is solved through the linear iterative method

- $x^{(0)}$ being given,

- for $m=0,1,2$, until convergence do

$$
x^{(m+1)}=M x^{(m)}+b .
$$

Clearly if these iterative methods converge, they will converge towards the solution $x$ of the following linear system

$$
(I-M) x=b
$$

where $I$ is the identity matrix. It is the above linear system that is solved by GMRES which only requires the right-hand side $b$ to be given and the way the matrix-vector $(I-M) x$ is performed. It is enough to observe that $b$ can be obtained by performing one iteration starting from $x^{(0)}=0$ and that $x^{(m)}-x^{(m+1)}=(I-M) x^{(m)}$ if the data of the linear problem are set to zero.

The first test concerns the case of a moderately elongated impenetrable domain corresponding to $L=4$ and the second, much more elongated, is obtained for $L=40$. Table 1 summarizes the numerical for each method in terms of accuracy and CPU time. For the iterative methods, we also compute the iteration number, noted "Iter" in Table 1 , obtained by reducing the residual by a factor $10^{-6}$. To measure the accuracy, we use the quantity $\mathscr{E}=\max \left|s(\theta)-s_{\mathrm{BE}}(\theta)\right|$ where $s_{\mathrm{BE}}(\theta)$ is the far field computed by the $\mathrm{BE}$ approach, known to be the less dispersive, using a very refined mesh of 30 points per wavelength. All the iterative methods were solved using the GMRES algorithm with a Krylov space of dimension 50, the underlying linear system being of order 1400 when coupling FEM with BEM. 


\begin{tabular}{|l|c|c|c|c|}
\hline & L & CPU & $\mathscr{E}$ & Iter \\
\hline \multirow{2}{*}{ BE } & 4 & 3.25 & - & - \\
& 40 & 153 & - & - \\
\hline \multirow{2}{*}{ FEBE } & 4 & 1.92 & 1.16 & - \\
& 40 & 63 & 0.63 & - \\
\hline \multirow{2}{*}{ FEBE2 } & 4 & 5.78 & 1.16 & - \\
& 40 & 79 & 0.81 & - \\
\hline \multirow{2}{*}{ LRC } & 4 & 8.53 & 1.19 & 9 \\
& 40 & 68 & 0.61 & 10 \\
\hline \multirow{2}{*}{ P-DDM } & 4 & 4.40 & 1.25 & 40 \\
& 40 & 512 & 1.01 & 63 \\
\hline
\end{tabular}

Table 1. Comparison of the various formulations in terms of accuracy, CPU time, and number of iterations.

The results reported in Table 1 confirm the robustness of the LRC formulation for the problems where the impenetrable zone is much larger than the one corresponding to the heterogeneous material. The number of iterations used by the LRC formulation to converge is almost independent of the size of the impenetrable zone. For $L=40$, only the FEBE formulation required a lower CPU time. It is worth noting, however, that the two-dimensional study given here is just of methodological interest.

Plots 6 and 7 show that the results provided by the LRC formulation completely agree with those of the other methods.

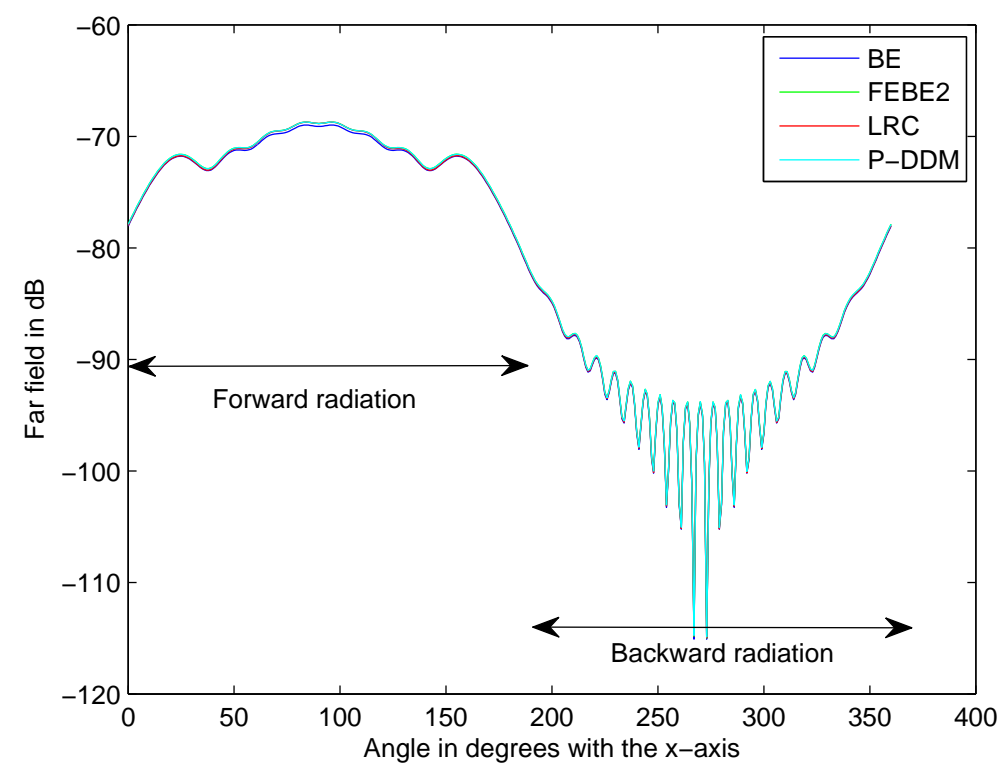

FIG. 6. Far field for $L=4$. 


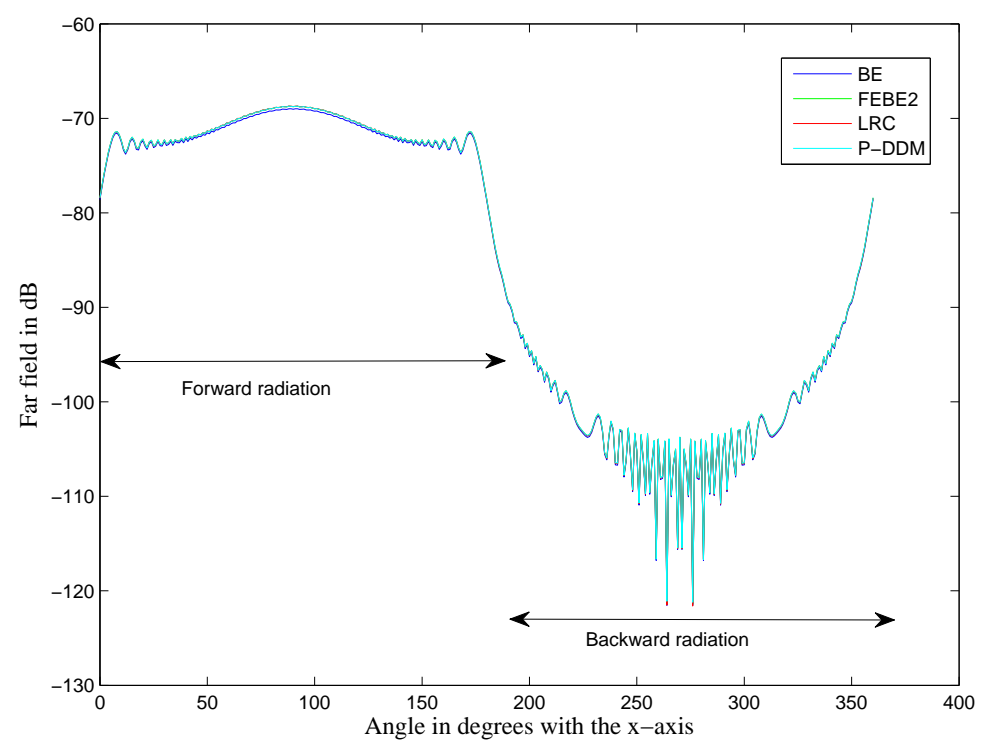

FIG. 7. Far field for $L=40$.

Finally, it is clear from the plots depicted in Fig. 8 and 9 that the LRC formulation as an iterative method outperforms the primal DDM approach.

\section{Concluding remarks}

We have designed a new coupling approach for solving wave propagation problems, where the computational domain is composed of a large impenetrable object on which is posed some penetrable material of relatively small size. The BEM is used to deal with the solution on the impenetrable surface, and the FEM approximates the solution in the heterogeneous region. We have established uniqueness and stability estimates and provide a theoretical basis to the method. Numerical results validate this approach, and have confirmed its effectiveness in practical computations.

However, some practical problems deserve further investigations. Indeed, the size of the elongated impenetrable zone requires particular treatment because of the corresponding dense matrix generated by the BEM. One way to tackle this problem resides in using a Krylov subspace iterative method coupled with an acceleration of the matrix-vector product by means of a Fast Multipole Method (cf., e.g., Chew et al. (2001); Fischer et al. (2004)) associated with a suitable preconditioning technique for the whole problem. A possible way is to use as a preconditioner an approximation of the Dirichlet-to-Neumann map, sometimes referred to as the On Surface Radiation Condition (cf. Antoine (2008)). Another approach regarding the design of a preconditioner consists of utilizing a thin layer of FE enclosing the boundary $\partial \Omega_{0}$ of the free propagation zone $\Omega_{0}$ and an accurate radiation condition on the truncating 


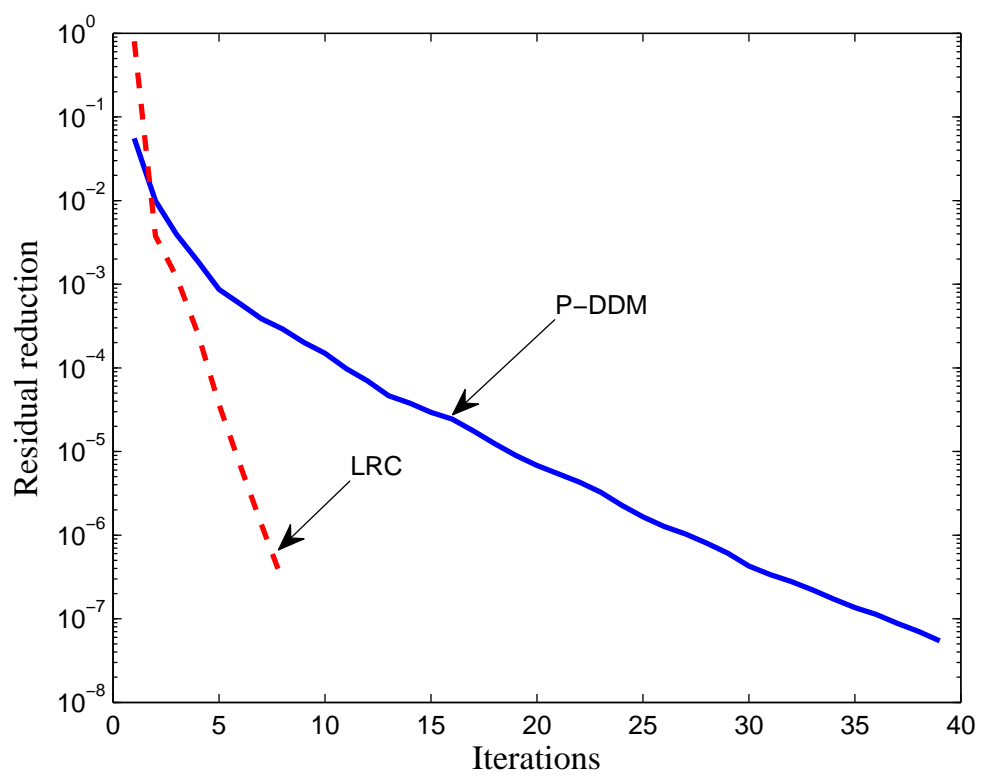

FIG. 8. Reduction of the residual for $L=4$

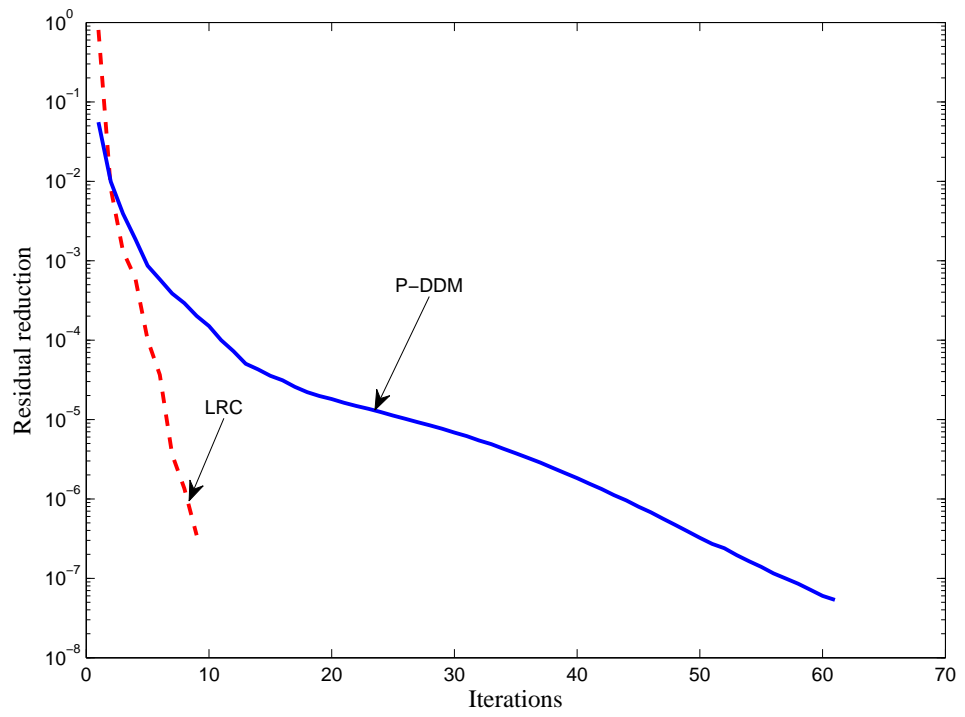

FIG. 9. Reduction of the residual for $L=40$. 
boundary (see Reiner et al. (2006)).

\section{Acknowledgments}

Yassine Boubendir gratefully acknowledges support from NSF through grant No. DMS-1016405.

\section{REFERENCES}

Alfonzetti, S., Borzì, G. \& SAlerno, N. (1998) Iteratively-improved Robin boundary conditions for the finite element solution of scattering problems in unbounded domains. Int. J. Numer. Meth. Engng, 42, 601-629.

Antolne, X. (2008) Advances in the on-surface radiation condition method: Theory, numerics and applications. Computational Methods for Acoustics Problems (F. Magoules ed.). Stirlingshire (Scottland): Saxe-Coburg Publications, pp. 207-232.

Ben Belgacem, F. (1999) The mortar finite element methodwith Lagrange multipliers. Numer. Math., 84, 173-197.

Ben Belgacem, F., Fournié, M., Gmati, N. \& Jelassi, F. (2005) On the Schwarz algorithms for the Elliptic Exterior Boundary Value Problems. M2AN. Mathematical Modelling and Numerical Analysis, 39, 693-714.

Ben Belgacem, F., Gmati, N. \& Jelassi, F. (2009) Convergence bounds of GMRES with Schwarz preconditioner for the scattering problem. Int. J. Numer. Meth. Engng., 80, 191-209.

Bendali, A., Boubendir, Y. \& FARes, M. (2007) A FETI-like domain decomposition method for coupling finite elements and boundary elements in large-size problems in acoustic scattering. Computers \& Structures, 85, 526-535.

Bendali, A. \& BoubendiR, Y. (2006) Non-overlapping domain decomposition method for a nodal finite element method. Numerisch Mathematik, 103, 515-537.

Bendali, A. \& FARES, M. (2008) Boundary integral equations methods in acoustics. Computer Methods for Acoustics Problems (F. Magoules ed.). Kippen, Stirlingshire, Scotland: Saxe-Coburg Publications, pp. 1-36.

Bendali, A. \& Souilah, M. (1994) Consistency estimates for the double-layer potential and application to the numerical analysis of the boundary element approximation of acoustic scattering by a penetrable object. Math. of Comp., 62, 65-91.

Boubendir, Y., Bendali, A. \& Fares, M. (2008) Coupling of a non-overlapping Domain Decomposition Method for a Nodal Finite Element Method with a Boundary Element Method. International Journal for Numerical Methods in Engineering, 73, 1624-1650.

Brezzi, F. \& Fortin, M. (1991) Mixed and Hybrid Finite Element Methods. New York: Springer-Verlag.

Buffa, A. \& Hiptmair, R. (2003) Galerkin boundary element methods for electromagnetic scattering. Topics in Computational Wave Propagation: Direct and Inverse Problems (M. A. et Al. ed.). Lecture Notes In Computational Science and Engineering, vol. 31. Berlin, Heidelberg, New York: Springer, pp. 83-124.

Chazarain, J. \& Piriou, A. (1982) Introduction to the Theory of Partial Differential Equations. Amsterdam and New York: North-Holland Publishing Co.

Chen, G. \& Zhou, J. (1992) Boundary Element Methods. London: Academic Press Inc.

Chew, W. C., JIN, J.-M., Michielssen, E. \& Song, J. (2001) Fast and Efficient Algorithms in Computational Electromagnetics. Norwood: Artech House, Inc.

Colton, D. \& Kress, R. (1983) Integral Equation Methods in Scattering Theory. New York: John Wiley and Sons.

Colton, D. \& Kress, R. (1992) Inverse Acoustic and Electromagnetic Scattering Theory. Series in Applied Mathematics, vol. 93. New York, Berlin, Heidelberg: Springer Verlag.

Costabel, M. (1988) A symmetric method for the coupling of finite elements and boundary elements. The Mathematics of Finite Elements and Applications, VI (Uxbridge, 1987). london: Academic Press, pp. 281288. 
24 of 24

Després, B. (1993) Domain decomposition method and the Helmholtz equation. II. Second International Conference on Mathematical and Numerical Aspects of Wave Propagation, Neuwark, Delaware. Philadelphia: SIAM, pp. 197-206.

Fischer, M., Gauger, U. \& Gaul, L. (2004) A multipole Galerkin boundary element method for acoustics. Engineering Analysis with Boundary Elements, 28, 155-162.

Gosselet, P. \& Rey, C. (2006) Non-overlapping domain decomposition methods in structural mechanics. Arch. Comput. Meth. Engng., 13, 515-572.

HAZARD, C. \& LENOIR, M. (1996) On the solution of time-harmonic scattering problems for Maxwell's equations. SIAM J. Math. Anal., 27, 1597-1630.

HSiAO, G. C. \& Wendland, W. L. (2008) Boundary Iintegral Equations. Berlin-Heidelberg: Springer.

JIN, J. (2002) The Finite Element Method in Electromagnetics, Second Edition. New York: John Wiley \& Sons.

Johnson, C. \& NÉDÉLEC, J.-C. (1980) On the coupling of boundary integral and finite element methods. Mathematics of Computation, 35, 1063-1079.

LENOIR, M. \& JAMI, A. (1978) A variational formulation for exterior problems in linear hydrodynamics. Comput. Meth. Appl. Mech. Engrg., 16, 341-359.

LI, Y. \& CENDES, Z. (1995) High-accuracy absorbing boundary conditions. IEEE Ttransactions on Magnetics, 31, 1524-1529.

LIU, J. \& JIN, J.-M. (2001) A novel hybridization of higher order finite element and boundary integral methods for electromagnetic scattering and radiation problems. IEEE Transactions on Antennas and Propagation, 49, 1794-1806.

Maischak, M., Mund, P. \& Stephan, E. P. (1997) Adaptive multilevel BEM for acoustic scattering. Comput. Methods Appl. Mech. Engrg., 150, 351-367.

MCLEAN, W. (2000) Strongly Elliptic Systems and Boundary Integral Equations. Cambridge, UK, and New York, USA: Cambridge University Press.

NÉDÉLEC, J.-C. (2001) Acoustic and Electromagnetic Equations: Integral Representations for Harmonic Problems. Berlin: Springer.

Reiner, R. C. J., DJellouli, R. \& Harari, I. (2006) The performance of local absorbing boundary conditionsfor acoustic scattering from elliptical shapes. Comput. Methods Appl. Mech. Engrg., 195, 3622-3665.

SAAD, Y. (1996) Iterative Methods for Sparse Linear Systems. Boston: PWS Pblishing Company.

Steinbach, O. (2003) Stability Estimates for Hybrid Coupled Domain Decomposition Methods. Berlin Heidelberg New York: Springer-Verlag.

TAYLOR, M. (1996) Partial Differential Equations I, Basic Theory. New York: Springer-Verlag.

VON PetersdorfF, T. (1989) Boundary integral equations for mixed Dirichlet, Neumann and trans-mission problems. Math. Meth. Appl. Sc., 11, 185-213.

WILCOX, C. H. (1975) Scattering theory for the d'Alembert equation in exterior domains, vol. 442. Berlin: Springer-Verlag.

Zerbib, N. (2006) Méthodes de sous-structuration et de décomposition de domaine pour la résolution des équations de maxwell : Application au rayonnement d'antenne dans un environnement complexe. Ph.D. thesis, National Institute for Applied Sciences (INSA), INSA Toulouse. 